\title{
Probing disk properties with open clusters
}

\author{
C. Bonatto, L. O. Kerber, E. Bica, and B. X. Santiago
}

Universidade Federal do Rio Grande do Sul, Instituto de Física, CP 15051, Porto Alegre 91501-970, RS, Brazil

e-mail: charles@if.ufrgs.br

Received 3 June 2005 / Accepted 19 September 2005

\begin{abstract}
We use the open clusters (OCs) with known parameters available in the WEBDA database and in recently published papers to derive properties related to the disk structure such as the thin-disk scale height, displacement of the Sun above the Galactic plane, scale length and the OC age-distribution function. The sample totals 654 OCs, consisting basically of Trumpler types I to III clusters whose spatial distribution traces out the local geometry of the Galaxy. We find that the population of OCs with ages younger than 200 Myr distributes in the disk following an exponential-decay profile with a scale height of $z_{\mathrm{h}}=48 \pm 3 \mathrm{pc}$. For the clusters with ages in the range $200 \mathrm{Myr}$ to $1 \mathrm{Gyr}$ we derive $z_{\mathrm{h}}=150 \pm 27 \mathrm{pc}$. Clusters older than $1 \mathrm{Gyr}$ distribute nearly uniformly in height from the plane so that no scale height can be derived from exponential fits. Considering clusters of all ages we obtain an average scale height of $z_{\mathrm{h}}=57 \pm 3$ pc. We confirm previous results that $z_{\mathrm{h}}$ increases with Galactocentric distance. The scale height implied by the OCs younger than $1 \mathrm{Gyr}$ outside the Solar circle is a factor $\sim 1.4-2$ larger than $z_{\mathrm{h}}$ of those interior to the Solar circle. We derive the displacement of the Sun above the Galactic plane as $z_{\odot}=14.8 \pm 2.4 \mathrm{pc}$, which agrees with previous determinations using stars. As a consequence of the completeness effects, the observed radial distribution of OCs with respect to Galactocentric distance does not follow the expected exponential profile, instead it falls off both for regions external to the Solar circle and more sharply towards the Galactic center. We simulate the effects of completeness assuming that the observed distribution of the number of OCs with a given number of stars above the background, measured in a restricted zone outside the Solar circle, is representative of the intrinsic distribution of OCs throughout the Galaxy. Two simulation models are considered in which the intrinsic number of observable stars are distributed: $(i)$ assuming the actual positions of the OCs in the sample, and (ii) random selection of OC positions. As a result we derive completeness-corrected radial distributions which agree with exponential disks throughout the observed Galactocentric distance range $5-14 \mathrm{kpc}$, with scale lengths in the range $R_{\mathrm{D}}=1.5-1.9 \mathrm{kpc}$, smaller than those inferred by means of stars. In particular we retrieve the expected exponential-disk radial profile for the highly depleted regions internal to the Solar circle. The smaller values of $R_{\mathrm{D}}$ may reflect intrinsic differences in the spatial distributions of OCs and stars. We derive a number-density of Solar-neighbourhood (with distances from the Sun $d_{\odot} \leq 1.3 \mathrm{kpc}$ ) OCs of $\rho_{\odot}=795 \pm 70 \mathrm{kpc}^{-3}$, which implies a total number of (Trumpler types I to III) OCs of $\sim 730$ of which $\sim 47 \%$ would already have been observed. Extrapolation of the completeness-corrected radial distributions down to the Galactic center indicates a total number of OCs in the range $(1.8-3.7) \times 10^{5}$. These estimates are upper-limits because they do not take into account depletion in the number of OCs by dynamical effects in the inner parts of the Galaxy. The observed and completeness-corrected age-distributions of the OCs can be fitted by a combination of two exponential-decay profiles which can be identified with the young and old OC populations, characterized by age scales of $\sim 100 \mathrm{Myr}$ and $\sim 1.9 \mathrm{Gyr}$, respectively. This rules out evolutionary scenarios based on constant star-formation and OC-disruption rates. Comparing the number of observed embedded clusters and candidates in the literature with the expected fraction of very young OCs, derived from the observed age-distribution function, we estimate that 3.4-8\% of the embedded clusters do actually emerge from the parent molecular clouds as OCs.
\end{abstract}

Key words. Galaxy: open clusters and associations: general - Galaxy: structure

\section{Introduction}

Understanding the true shape of the Milky Way is a challenging task which depends essentially on the availability of as complete as possible Galactic surveys of the spatial distribution of stars, star clusters, H I gas, molecular clouds, etc. These observations can be used to better constrain theoretical mass models of the Galaxy. The modern view of the Milky Way pictures it as composed basically of the center and nuclear bulge, a thin disk hosting most of the OCs, a thick disk containing the old OCs, and the extended halo hosting the old stars, white dwarfs and globular clusters (e.g. Majewski 1993). The gas and stellar density of the disk is usually expressed as a combination of exponential-decay profiles for the horizontal and vertical directions, $\rho(r, z) \propto \mathrm{e}^{-\left(r / R_{\mathrm{D}}\right)} \mathrm{e}^{-\left(|z| / z_{\mathrm{h}}\right)}$, where $R_{\mathrm{D}}$ is the scale length and $z_{\mathrm{h}}$ the scale height (e.g. Binney \& Tremaine 1987).

The kinematics and spatial distribution of large numbers of stars have been the best used probes of the Galaxy structure. For instance, $\mathrm{M}$ dwarfs are found to distribute vertically with relatively large scale heights, $z_{\mathrm{h}} \sim 300 \mathrm{pc}$, in contrast to the younger A-type stars with $z_{\mathrm{h}} \sim 100 \mathrm{pc}$ (Faber et al. 1976). 
Giants and subgiants have scale heights typically between 200 and $500 \mathrm{pc}$, though the uncertainty is larger than that of the dwarf luminosity class (e.g. Bahcall \& Soneira 1981). Using star counts from the Sloan Digital Sky Survey taken from two high-latitude $\left(49^{\circ}<|b|<64^{\circ}\right)$ samples north and south of the Galactic plane Chen et al. (2001) derived $z_{\mathrm{h}}=330 \pm 3 \mathrm{pc}$ for the old thin disk and $z_{\mathrm{h}}=580-750 \mathrm{pc}$ for the thick disk. They suggest that the thick disk formed through heating of a preexisting thin disk caused by the merging of a satellite galaxy. Based on the kinematics of sdB stars de Boer et al. (1997) and Altmann et al. (2004) found that these stars are characterized by two distributions, a disk one with $z_{\mathrm{h}} \sim 1 \mathrm{kpc}$ and an extended one with $z_{\mathrm{h}} \sim 7 \mathrm{kpc}$. In recent years considerable amounts of data on parallax and proper motion of stars has allowed to probe in detail the Galactic structure in distances of up to $2 \mathrm{kpc}$ from the Sun. In particular Kaempf et al. (2005) using the kinematics and spatial distribution of red horizontal-branch stars selected from the HIPPARCOS catalogue derived a disk scale height of $z_{\mathrm{h}} \approx 0.6 \mathrm{kpc}$.

With respect to the vertical position of the Sun $\left(z_{\odot}\right)$, asymmetries in star counts above and below the Galactic plane have put it displaced a few parsecs to the North. Several methods used in the past constrained the value of $z_{\odot}$ to the range $4-40 \mathrm{pc}$ (see, e.g. the account in Cohen 1995). More recently, different sets of data have been used to obtain more accurate values of $z_{\odot}$. Cohen (1995), using IRAS point-source counts at 12 and $25 \mu \mathrm{m}$ obtained $z_{\odot}=15.5 \pm 0.7 \mathrm{pc}$. Hammersley et al. (1995) examined star counts and surface brightness maps from COBE, IRAS and the Two-micron Galactic Survey to derive $z_{\odot}=15.5 \pm 3 \mathrm{pc}$. The high-Galactic latitude data of Chen et al. (2001) resulted in $z_{\odot}=27 \pm 4$ pc.

Measurements of the horizontal scale length by means of stars place $R_{\mathrm{D}}$ in the range from 2.2 to $3.5 \mathrm{kpc}$ (de Vaucouleurs $\&$ Pence 1978; Knapp et al. 1978; McCuskey 1969).

Open clusters are formed in, and distribute throughout the disk. Interactions with the disk and the tidal pull of the Galactic center/bulge tend to destroy the poorly-populated OCs in a time-scale of a few $10^{8} \mathrm{Myr}$ (Bergond et al. 2001). The dynamical disruption is more critical particularly for the OCs more centrally located. The survivors, however, may reach large vertical distances from the plane spanning the thick disk. Although the number of known OCs is small compared to stars, it is relatively simple and accurate to derive distance and age for these objects. In this sense, OCs - in particular their spatial distribution - may serve as a direct probe of the disk structure. By means of a sample of 72 OCs Janes \& Phelps (1994) found that the young OCs (age $\leq 800 \mathrm{Myr}$ ) are distributed almost symmetrically about the Sun with a scale height perpendicular to the Galactic plane of $z_{\mathrm{h}} \approx 55 \mathrm{pc}$, while for the old OCs they found $z_{\mathrm{h}} \approx 375 \mathrm{pc}$.

It is interesting to point out that since Janes \& Adler (1982) study the number of OCs with derived parameters increased nearly $50 \%$, taking as reference the clusters currently with parameters in the WEBDA ${ }^{1}$ OC database (Mermilliod 1996). However, in the recent revision of OCs Dias et al. (2002) report a total of 1537 catalogued OCs. This clearly indicates the need

\footnotetext{
${ }^{1}$ http://obswww . unige.ch/webda
}

to explore more systematically the available OC databases. WEBDA gathers data from a variety of sources, which implies different observational instrumentation and analysis methods and, consequently uncertainties in the values of parameters. We deal with the non-uniformity of the parameters by $(a)$ working with bins wide enough to take into account most of the uncertainties, and $(b)$ assuming $1 \sigma$ Poisson errors to assign statistical significances to the results.

In this paper we build an OC sample composed of the WEBDA objects added to 10 other OCs studied in recent papers. We intend to use this OC sample, particularly the $z$-measurements, to derive the disk scale height $\left(z_{\mathrm{h}}\right)$, the displacement of the Sun above the Galactic plane $\left(z_{\odot}\right)$, and the horizontal scale length. In addition, we use the present OC sample to study statistical properties related to the reddening, distance from the Sun, location in the Galaxy, completeness and, particularly, to derive the intrinsic OC age-distribution function.

Previous statistical works on the relation of OC parameters with Galactic structure are, e.g. Lyngå (1982), Janes \& Phelps (1994), Nilakshi et al. (2002), and Tadross et al. (2002). The main results of Lyngå (1982), related to the present work, are (i) a correlation of cluster age with Galactocentric distance; (ii) an exponential OC disintegration time scale of $\sim 10^{8} \mathrm{yr}$; (iii) displacement of the Sun over the Galactic plane of $20 \mathrm{pc}$; and (iv) a $z$-distribution which differs only for the oldest clusters. Nilakshi et al. (2002) found that OCs with Galactocentric distances larger than $9.5 \mathrm{kpc}$ have larger sizes. Tadross et al. (2002) found ( $i$ ) evidence that older clusters are found preferentially in the outer parts of the disk; and (ii) that most of the OCs younger than $32 \mathrm{Myr}$ are concentrated on the Perseus arm.

This paper is organized as follows. In Sect. 2 we present the OC sample and discuss statistical properties related to the spatial and age distributions. In Sect. 3 we discuss qualitative aspects affecting sample completeness. In Sect. 4 we derive the displacement of the Sun above the Galactic plane and the observed scale height. In Sect. 5 we simulate the effects of sample completeness. In Sect. 6 we derive completeness-corrected radial and vertical distribution functions, and discuss properties of completeness-corrected age distribution functions. In Sect. 7 inferences on the total number of OCs in the Galaxy are presented. Concluding remarks are in Sect. 8.

\section{Statistical characterization of the $O C$ sample}

The OCs used in the present study are those with Galactic coordinates, distance from the Sun, reddening and age catalogued in WEBDA. To these we add 10 recently studied OCs (2 in Bica et al. 2003c; 3 in Bonatto et al. 2004; and 5 in Ortolani et al. 2005). The sample totals 654 OCs with known distance from the Sun (of which 636 have age determination).

The significant number of OCs in this sample presents an opportunity to study properties of clusters in different age ranges separately. We selected the age ranges $(i)$ age $\leq$ $200 \mathrm{Myr}$ (young OC population); (ii) $200 \mathrm{Myr} \leq$ age $\leq 1 \mathrm{Gyr}$ (moderate-age OCs); and (iii) age $\geq 1$ Gyr (old OCs) both for statistical and population representativity purposes. The number of clusters in each age range is 402 (young), 148 (moderateage) and 86 (old). 


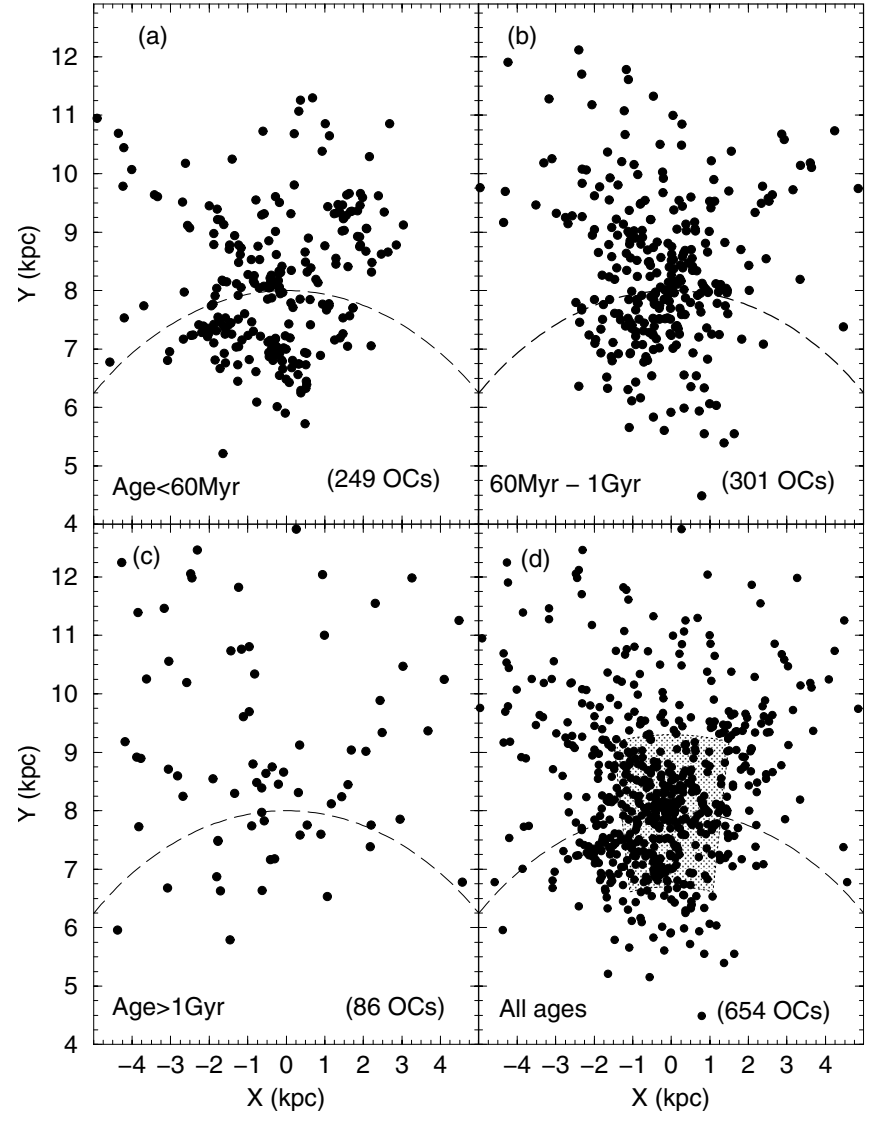

Fig. 1. Spatial distribution of the OCs, according to age groups. The Solar circle is shown by the dashed line, and the Galactic center is located at $(0,0)$. Shaded area in panel $\mathbf{d})$ : region encompassed by the restricted sample (Sect. 7). For comparison purposes the coordinate definitions follow Janes \& Phelps (1994).

\subsection{Spatial distribution}

In Fig. 1 we show the spatial distribution of the OCs in the present sample projected onto the $X$ and $Y$ plane, separated according to age groups. Throughout this paper we use $8.0 \mathrm{kpc}$ (Reid 1993) as the distance of the Sun to the Galactic center. In this figure conspicuous structures of the local disk are apparent. For clarity we show the Solar circle in all panels. Note that in panels $\mathrm{a}$ and $\mathrm{b}$ the threshold between young and moderate ages has been changed to $60 \mathrm{Myr}$ in order to better isolate OCs related to spiral arms. OCs younger than $60 \mathrm{Myr}$ (panel a) trace out the local distribution of the Orion Arm (Georgelin \& Georgelin 1970), in which the Sun is located close to the inner border. Another conspicuous feature defined by the young OCs is the gap towards the Galactic center at $d_{\mathrm{GC}} \approx 7.5 \mathrm{kpc}$ related to the dip before the Sgr-Car Arm. Clusters older than $1 \mathrm{Gyr}$ (panel c) are uniformly distributed but with an asymmetry in the number of OCs towards the anti-center, a region where they are well-known to populate (Friel 1995). The all-ages sample is shown in panel $\mathrm{d}$ where traces of the Orion Arm, the gap at $d_{\mathrm{GC}} \approx 7.5 \mathrm{kpc}$ and the radial asymmetry can still be detected.

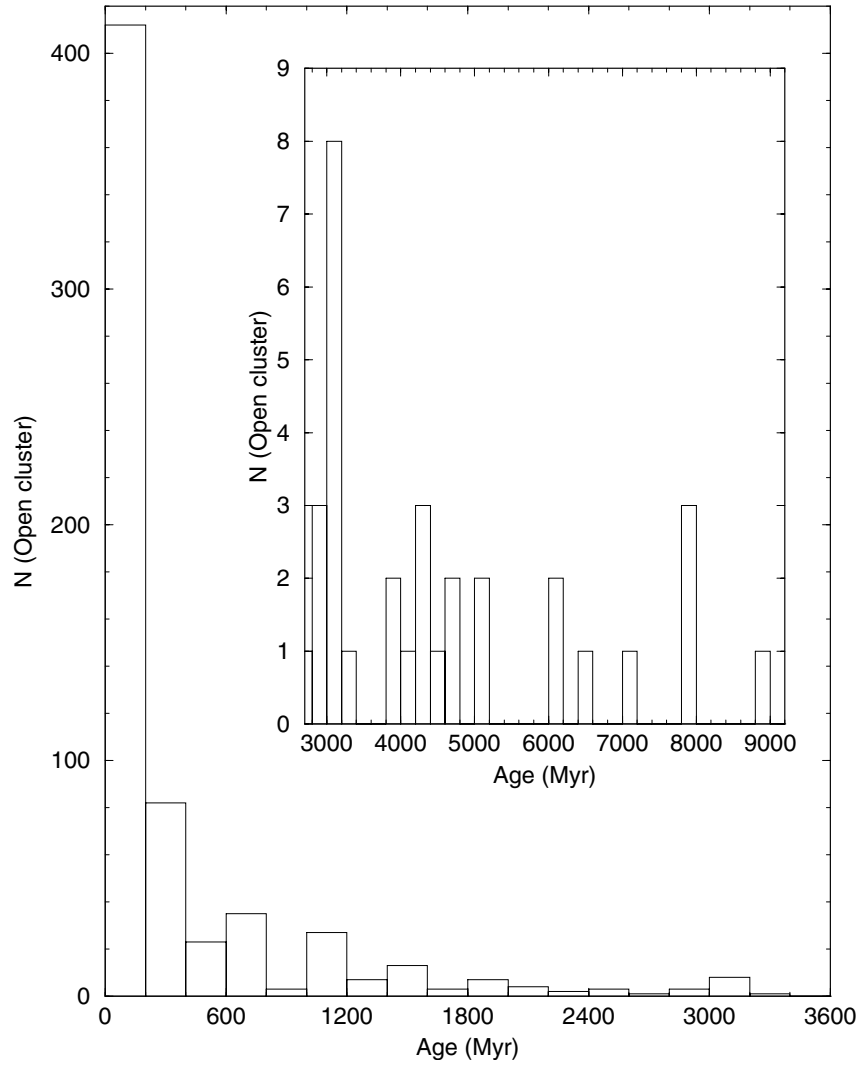

Fig. 2. Age histogram of the OC sample for age $\leq 3800$ Myr. Peaks occur at $\sim 100 \mathrm{Myr}, \sim 700 \mathrm{Myr}$, and $\sim 3.1 \mathrm{Gyr}$. The inset shows the histogram for age $\geq 2800 \mathrm{Myr}$.

\subsection{Observed age histogram}

The observed age histogram of the present OCs is shown in Fig. 2 with bins of $200 \mathrm{Myr}$ which are wide enough to accommodate most of the uncertainties related to the different age-determination techniques included in the WEBDA sample. Two conspicuous peaks occur at $\sim 100 \mathrm{Myr}$ (characterizing the young OCs) and $\sim 700 \mathrm{Myr}$ (moderate-age OCs). A lesspronounced peak occurs at $\sim 3.1 \mathrm{Gyr}$, which can be associated to the old OC distribution.

The age-distribution function will be presented and discussed in Sect. 6.5.

\subsection{Galactic coordinates}

In the left panels of Fig. 3 we examine the distribution of OCs in terms of Galactic longitude. In all age ranges the number of OCs drops off towards the Galactic center and increases roughly towards the third quadrant. These facts might reflect both an observational limitation in the sense that lowcontrast clusters are more difficult to detect in directions projected against the Galaxy center/bulge (or more simply, observers tended to avoid crowded central fields - Sect. 3) and the enhanced tidal-disruption probability of OCs closer to the Galactic center/bulge (Bergond et al. 2001).

The distribution of OCs in terms of Galactic latitude is in the right panels of Fig. 3. As expected, most of the OCs are tightly concentrated close to the Galactic plane (panel e). 

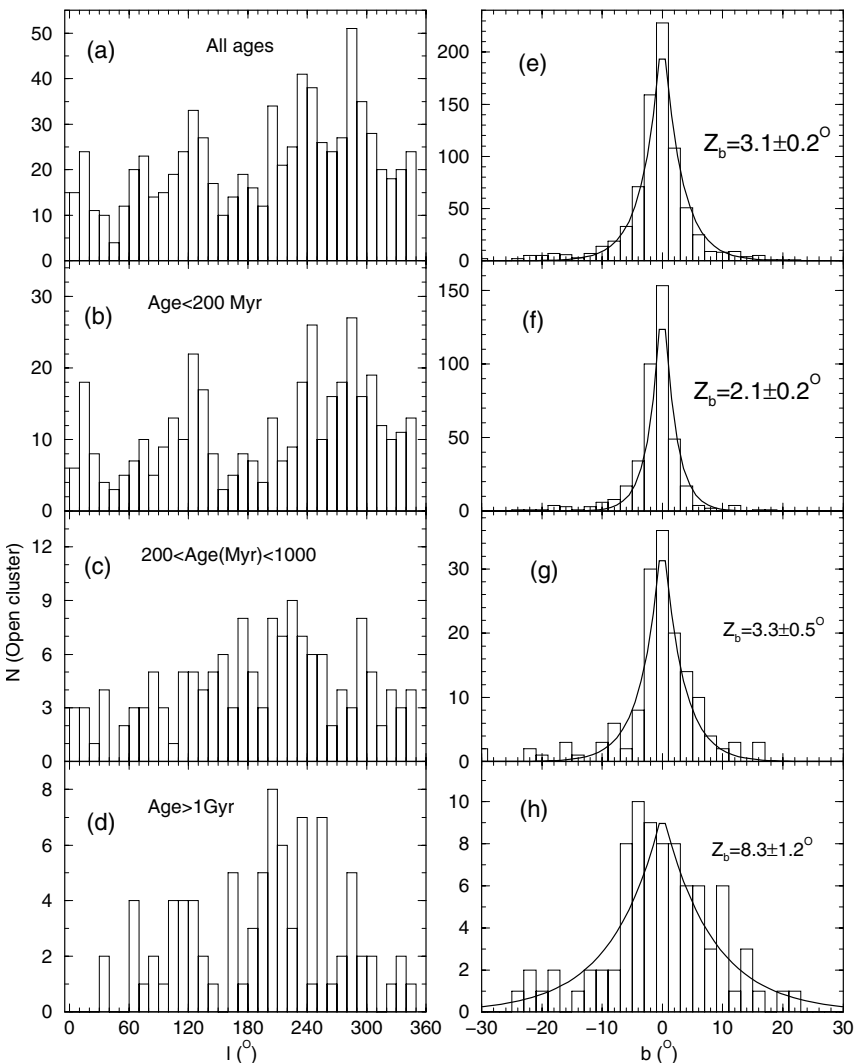

Fig. 3. Distribution of OCs in terms of Galactic longitude (left panels) and latitude (right panels). Solid lines: least-squares fit with the function $N \propto \mathrm{e}^{-\left|b / z_{b}\right|}$. Panels a) and e): OCs of all ages. Panels b) and f): OCs younger than $200 \mathrm{Myr}$. Panels c) and g): OCs with age in the range 200 Myr-1 Gyr. Panels d) and h): OCs older than 1 Gyr.

However, the distributions become wider for older age groups (panels $\mathrm{f}$ to $\mathrm{h}$ ). The distributions can be analytically described by the exponential-decay function $N \propto \mathrm{e}^{-\left|b / z_{b}\right|}$. We find $z_{\mathrm{b}}=$ $2.10 \pm 0.20^{\circ}$ for the young OCs (panel f), $z_{\mathrm{b}}=3.47 \pm 0.51^{\circ}$ for the moderate-age OCs (panel g), and $z_{\mathrm{b}}=8.27 \pm 1.13^{\circ}$ for the old OCs (panel h). The all-ages distribution (panel e) is characterized by $z_{\mathfrak{b}}=3.14 \pm 0.21^{\circ}$. The asymmetry observed in the old-age distribution with respect to Galactic latitude (panel h) may be accounted for by completeness (Sects. 3 and 5). Because these clusters are not as concentrated near the Galactic plane as the young OCs (Fig. 7), the old OCs located above the Galactic plane become easier to detect than the ones in the opposite side. A similar, but less-conspicuous effect can be seen in the distribution of the moderate-age OCs (panel g). Alternatively, this asymmetry might reflect an intrinsic difference in the distribution of old OCs above and below the plane.

\subsection{Distance from the Sun and reddening}

In the left panels of Fig. 4 we show the distribution of OCs with respect to the distance from the Sun, in bins of $0.2 \mathrm{kpc}$. The young (panel b) and moderate-age (panel c) OCs present similar distributions with peaks at $d_{\odot} \approx 1.7 \mathrm{kpc}$ and $d_{\odot} \approx 1.3 \mathrm{kpc}$, respectively. Observational limitations probably affect the
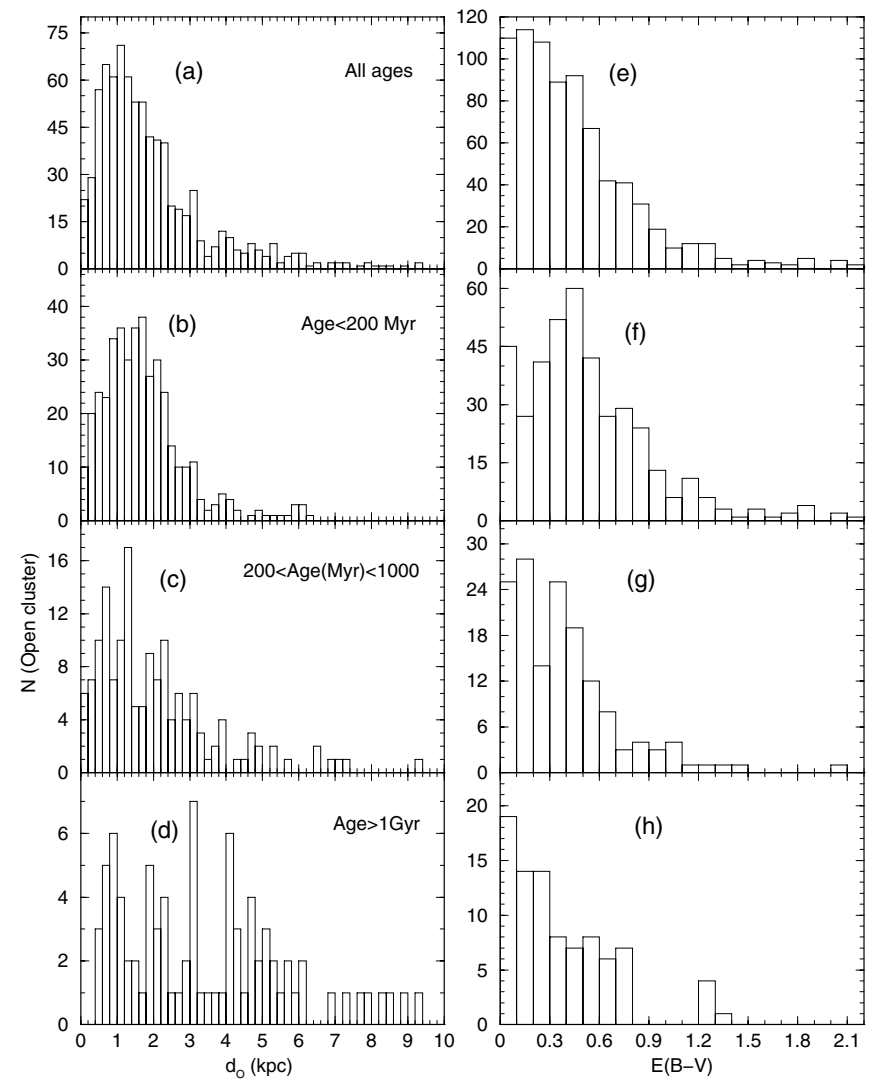

Fig. 4. Distribution of OCs in terms of distance from the Sun (left panels) and reddening (right panels). Panel ages as in Fig. 3.

detection of low-contrast OCs for $d_{\odot} \geq 1.7 \mathrm{kpc}$. The old OCs (panel d) are more evenly distributed (Fig. 1, panel d).

With respect to the reddening (right panels of Fig. 4) the distribution of the young OCs (panel $f$ ) is wider than those of the moderate-age (panel g) and old (panel h) OCs. This fact probably can be accounted for by more significant internal reddening affecting young OCs. In addition, excess interstellar reddening also affects more significantly young OCs, since these clusters are more tightly concentrated to the Galactic plane (panel $\mathrm{f}$ in Fig. 3). The strong dependence of reddening on OC location in the Galaxy can be appreciated in panels a and $b$ of Fig. 5. As expected, the reddening increases markedly towards the Galactic center/bulge (1st and 4th quadrants in panel a) and plane (the conspicuous peak in panel b). $E(B-V)$ increases nearly linearly with the distance from the Sun up to $\approx 3 \mathrm{kpc}$ (panel c); the scatter increases significantly for larger distances. The excess reddening observed in the young OCs is apparent as well in panel d.

\subsection{Galactocentric distance}

In Fig. 6 we examine histograms with the number of observed OCs in bins of Galactocentric distance. Similarly to the distance from the Sun, the old OCs (panel d) are more evenly distributed than the young (panel b) and moderate-age (panel c) OCs. The radial distribution of the all-ages OCs (panel a) is not symmetrical about the Galactocentric distance of the Sun, instead it presents a maximum at the Solar position and drops 

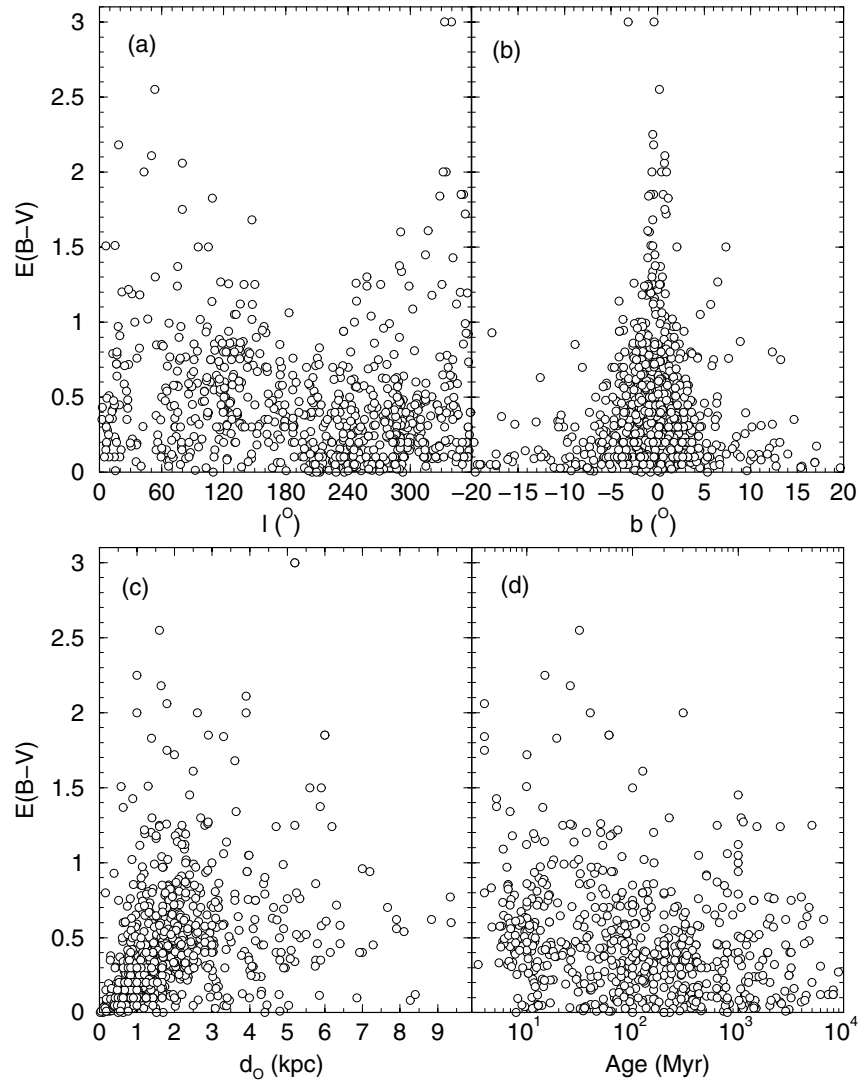

Fig. 5. Dependence of reddening on Galactic longitude (panel a)), latitude (panel b)), distance from the Sun (panel c)) and cluster age (panel d)). Each point represents an OC.

off more sharply towards the Galactic center than outside the Solar circle. OCs younger than $200 \mathrm{Myr}$ and those with age in the range $200 \mathrm{Myr}-1 \mathrm{Gyr}$ present similar radial distributions. The observed radial distribution of the OCs clearly does not resemble that expected of an exponential disk (e.g. Binney \& Tremaine 1987). We will return to this point in Sect. 7.

\section{Qualitative considerations on sample completeness}

In general terms, cluster detection-rates depend on factors such as the stellar content (both in absolute numbers and the presence of giants), apparent magnitude at the turnoff, distance from the Sun, stellar background projected onto the OC direction, etc. External factors such as systematic surveys with deep photometry are necessary as well to detect as many OCs as possible. In addition, the identification of a stellar concentration as a star cluster depends on the analysis of the colour-magnitude diagram morphology, the structure of radial-density profiles, and proper-motion data, when available (e.g. Bonatto \& Bica 2005; Bica \& Bonatto 2005a,b, and references therein).

The present sample is not complete, particularly with respect to OCs at large distances from the Sun and/or lowcontrast clusters. The cluster data in WEBDA come from photographic, photoelectric or CCD photometry taken with different instrumentation over the last 50 years. This heterogeneity of data sources precludes objective inferences on the

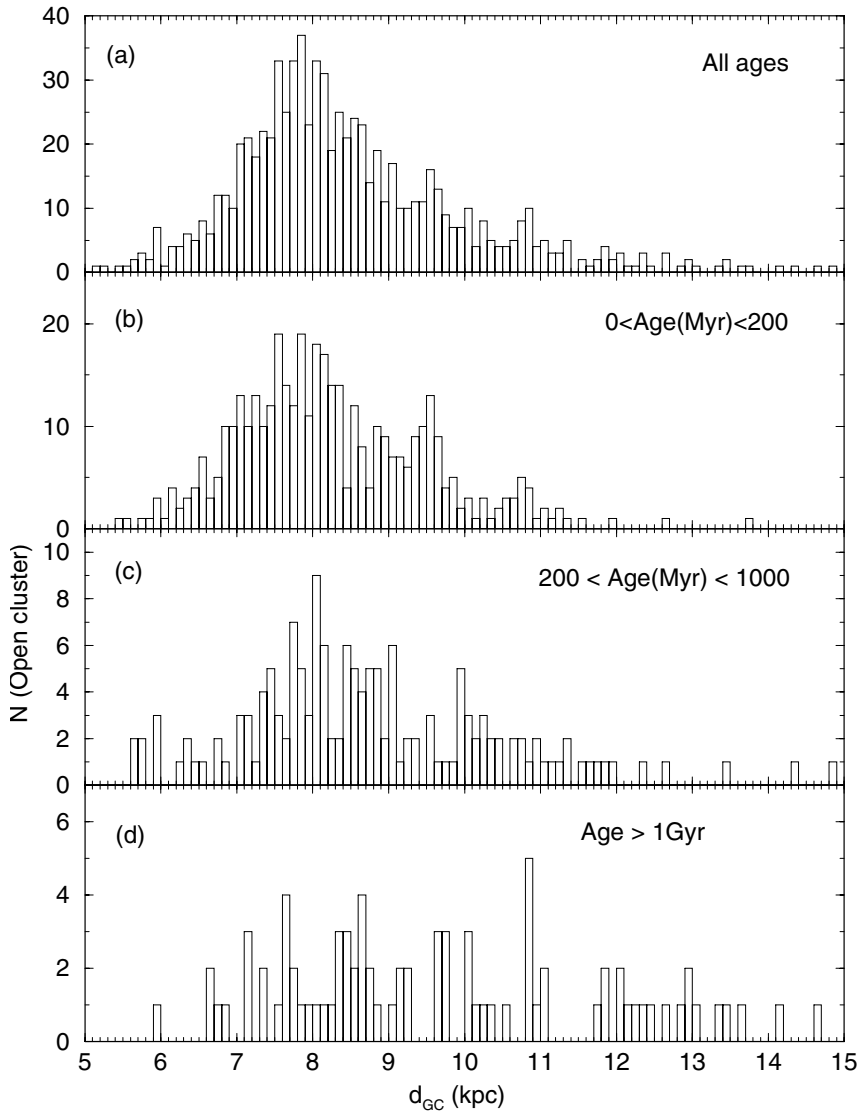

Fig. 6. Distribution of OCs in terms of Galactocentric distance. Panel ages as in Fig. 3.

faint magnitude-limit of the stars, total number of stars, surface brightness or density contrast in the present OCs. Basically the WEBDA sample contains OCs of the denser Trumpler types I to III (Ruprecht 1966), because observers naturally directed their attention to this kind of object.

The following argument illustrates the difficulties associated with the identification of low-contrast clusters in different regions of the Galaxy. Assume an OC whose radial density profile can be described by the two-parameter King (1966) law, $\sigma(r)=\sigma_{\mathrm{g}}+\frac{\sigma_{0 \mathrm{~K}}}{1+\left(r / R_{\mathrm{c}}\right)^{2}}$, where $\sigma_{\mathrm{bg}}$ is the background surface density of stars, $\sigma_{0 \mathrm{~K}}$ is the central density of stars (above the background), and $R_{\mathrm{c}}$ is the core radius. In fact, the background represents the contamination of non-cluster stars projected onto the OC angular area, integrated along the direction towards the OC, fore and background stars alike. In this sense the background density of an OC is a function of $\ell$ and $b$ only, and not of distance from the Sun (Sect. 5).

We define the contrast parameter as $\delta_{\mathrm{c}}=\frac{\sigma(0)}{\sigma_{\mathrm{bg}}}=1+\frac{\sigma_{0 \mathrm{~K}}}{\sigma_{\mathrm{bg}}}$. Integration of $\sigma(r)$ from the center to the limiting radius $\left(R_{\lim }\right)$ and considering that $R_{\lim } \geq 5 \times R_{\mathrm{c}}$ (Bonatto \& Bica 2005), the total number of cluster stars (background-subtracted) is $N * \approx 2 \pi \sigma_{0 \mathrm{~K}} R_{\mathrm{c}}^{2} \ln \left(R_{\lim } / R_{\mathrm{c}}\right)$. Thus, the contrast parameter as a function of the background density and effective number of stars can be expressed as

$\delta_{\mathrm{c}} \approx 1+\frac{\left(N * / \sigma_{\mathrm{bg}}\right)}{2 \pi \ln \left(R_{\lim } / R_{\mathrm{c}}\right) R_{\mathrm{c}}^{2}}$ 
Extractions of $2 \mathrm{MASS}^{2}$ photometry in directions which do not intercept detected clusters show that the average background stellar density in low-latitude $\left(|b| \leq 5^{\circ}\right)$ regions towards the Galactic center/bulge is a factor $\sim 10$ as large as that towards the anti-center (Sect. 5). Thus, an anti-center cluster with $\delta_{\mathrm{c}} \sim 11$ would have $\delta_{\mathrm{c}} \sim 2$ when projected against the center/bulge. In this sense, if the typical detection limit of WEBDA OCs is restricted to, say $\delta_{\mathrm{c}} \geq 2$, surveys towards the Galactic center/bulge would miss the whole class of OCs with $\delta_{\mathrm{c}}$ in the range 2 to 11 which in principle would be detected towards the anti-center.

To give an idea of the values $\delta_{\mathrm{c}}$ takes on with actual OCs we quote here some examples taken from Bonatto \& Bica (2005) and Bica \& Bonatto (2005b), derived with 2MASS data. The high-Galactic latitude OCs NGC $188\left(b=+22.39^{\circ} ; d_{\odot} \sim\right.$ $\left.1.7 \mathrm{kpc} ; M \sim 3800 M_{\odot}\right)$ and $\mathrm{M} 67\left(b=+31.89^{\circ} ; d_{\odot} \sim 0.9 \mathrm{kpc}\right.$; $\left.M \sim 990 M_{\odot}\right)$ present $\delta_{\mathrm{c}}=22.5 \pm 2.9$ and $\delta_{\mathrm{c}}=34.7 \pm 5.7$, respectively, while the populous and rather low-latitude cluster NGC $2477\left(b=-5.82^{\circ} ; d_{\odot} \sim 1.2 \mathrm{kpc} ; M \sim 5300 M_{\odot}\right)$ has $\delta_{\mathrm{c}}=20.7 \pm 1.9$. On the other hand, the relatively lowcontrast, third-quadrant OCs Ruprecht $78\left(b=-1.88^{\circ} ; d_{\odot} \sim\right.$ $\left.1.3 \mathrm{kpc} ; M \sim 860 M_{\odot}\right)$, Haffner $4\left(b=-3.62^{\circ} ; d_{\odot} \sim 1.7 \mathrm{kpc}\right.$; $\left.M \sim 260 M_{\odot}\right)$ and Trumpler $13\left(b=-2.34^{\circ} ; d_{\odot} \sim 1.9 \mathrm{kpc}\right.$; $\left.M \sim 420 M_{\odot}\right)$, present $\delta_{\mathrm{c}}=4.2 \pm 2.6, \delta_{\mathrm{c}}=4.3 \pm 2.2$, and $\delta_{\mathrm{c}}=8.9 \pm 5.5$, respectively.

Completeness is as well affected by cluster distance from the Sun in depth-limited photometry. OC stars fainter than some apparent magnitude may present exceedingly large photometric errors and consequently, end up not being separated from the background. To estimate the dependence of this effect on distance from the Sun we consider an artificial OC characterized by a standard Salpeter (Salpeter 1955) mass function $\left(\phi(m) \propto m^{-(1+\chi)}\right.$, with $\left.\chi=1.35\right)$ with the turnoff at $m=5 M_{\odot}$. Based on our previous experience in the analysis of OCs with 2MASS data (e.g. Bonatto \& Bica 2005, and references therein) we take stars with $J=15$ as representative of the average faint-magnitude limit which still can be used to derive OC structural parameters. Using the mass-luminosity relation derived from the $10 \mathrm{Myr}$, Solar-metallicity Padova isochrone (Girardi et al. 2002), the low-mass limit corresponding to $J=15$ can be expressed as a function of distance from the Sun as $m_{\text {low }}\left(d_{\odot}\right)=-1.18+0.6 \log \left(d_{\odot}\right)+3.4 \times 10^{-6} d_{\odot}{ }^{3 / 2}$. Integration of the mass function from the low-mass limit to the turnoff yields an estimate of the effective number of stars above the background, $N *$. To a good approximation, this number can be expressed as a function of distance from the Sun (for $\left.0.2 \leq d_{\odot}(\mathrm{kpc}) \leq 6\right)$ as $N *\left(d_{\odot}\right) \propto\left(d_{\odot} / 1 \mathrm{kpc}\right)^{-1}$. Thus, a given OC placed twice as distant from the Sun ends up presenting only about half of the stars above the background. Roughly speaking, this effect is similar to doubling the value of $\sigma_{\mathrm{bg}}$ in Eq. (1), which in turn produces a smaller value of $\delta_{\mathrm{c}}$.

From the observational point of view, recent proper-motion and parallax data have been used to explore the low-contrast end (even less concentrated than Trumpler type IV) of the

\footnotetext{
2 The Two Micron All Sky Survey, All Sky data release (Skrutskie et al. 1997), http://www.ipac.caltech.edu/2mass/ releases/allsky/
}

OC distribution. An example is the use of HIPPARCOS data to search for poorly-populated OCs or candidates in the solarvicinity $\left(d_{\odot} \leq 500 \mathrm{pc}\right)$ by Platais et al. (1998) which yielded about 10 such objects which have large angular sizes. One must be aware of the completeness effects discussed above, but it is also interesting to point out that at least for the Solar-vicinity the number of extremely-low contrast OCs appears not to be exceedingly large.

We conclude from the above that the present sample (and the results derived therefrom) - although certainly affected by completeness - can be taken as statistically representative of OCs of Trumpler types I to III.

The discussions above raise important points related to sample completeness and make it clear the need to undertake efforts to survey more thoroughly with deeper photometry - at least the Solar neighbourhood - to complete the local census of the OCs, particularly the low-contrast ones. However, they alone do not allow us to objectively quantify the completeness either of a particular OC or the average completeness of the whole sample. We will return to this issue in Sect. 5 where the quantitative effects of completeness will be derived by means of simulation.

\section{The observed disk scale height}

We calculated the vertical position $z$ of all OCs with Galactic longitude and latitude, and distance from the Sun. The resulting distributions are shown in Fig. 7 for the different age groups. The uncertainties correspond to $1 \sigma$ Poisson errors. Similarly to the Galactic latitude (Fig. 3), the $z$-distributions also depend on OC age, in the sense that older OCs are more evenly distributed in $z$ than the young OCs. The young (panel b) and moderate-age (panel c) OCs have distributions following exponential-decay profiles, while the old OCs (panel d) are almost uniformly distributed in height. The total number of OCs in each distribution is 402 (young), 148 (moderate-age) and 86 (old). Note that the number of clusters with $|z| \geq 400 \mathrm{pc}$, which are not shown in Fig. 7, is 0 for the young, 12 for the moderate-age and 27 for the old OCs.

Thus, the near uniformity in the z-distribution of the old OCs cannot be accounted for by small-number statistics alone, but it appears to reflect their intrinsic distribution.

In order to reach a higher $z$-resolution we built the all-ages distribution (panel a) with bins of $5 \mathrm{pc}$, while for the remaining distributions the $z$-bin is $10 \mathrm{pc}$. The higher $z$-resolution is necessary in order to derive more accurately the distance of the Sun from the Galactic plane while at the same time keeping the $1 \sigma$ Poisson errors non-prohibitively large.

We fit the all-ages distribution in panel a with the function $\phi(z)=\phi_{0} \mathrm{e}^{-\left|\left(z+z_{\odot}\right) / z_{\mathrm{h}}\right|}$, where $z_{\odot}$ is the displacement of the Sun with respect to the Galactic plane and $z_{\mathrm{h}}$ is the vertical scale height. For this particular analysis we center the coordinate system on the Sun. We obtain $z_{\odot}=14.8 \pm 2.3 \mathrm{pc}$ (above the plane), which within the uncertainties, is in excellent agreement with the values calculated by Cohen (1995) and Hammersley et al. (1995) using stars. This value of $z_{\odot}$ was kept fixed when fitting the young and moderate-age OCs distributions. The results are in Table 1. It is remarkable that large 


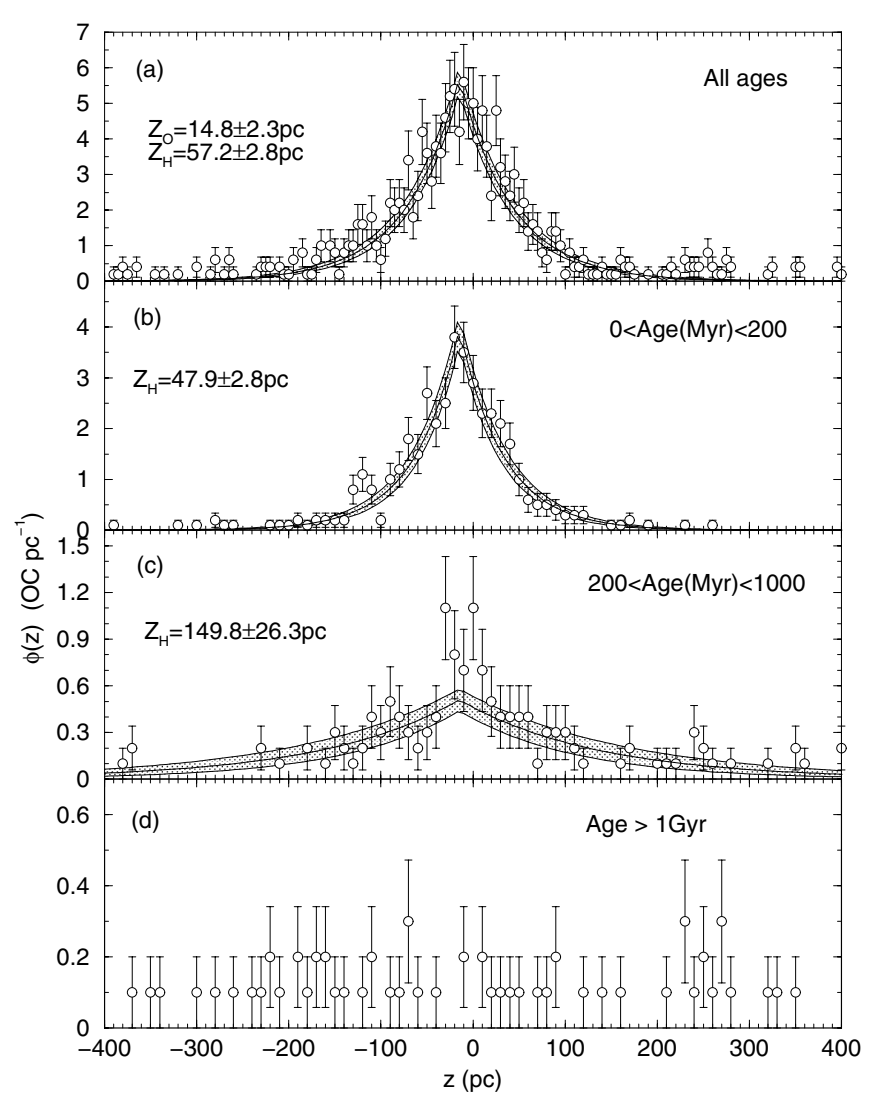

Fig. 7. Distribution of OCs in terms of height over the Galactic plane. Age groups are considered separately. Solid lines: least-squares fit with $\phi(z)=\phi_{0} \mathrm{e}^{-\left|\left(z+z_{\odot}\right) / z_{\mathrm{h}}\right|}$. Shaded areas: $1 \sigma$-standard deviation. Top panel: bins of $5 \mathrm{pc}$ in $z$. Bottom panels: $10 \mathrm{pc}$ bins.

Table 1. Observed disk-structural parameters.

\begin{tabular}{ccccc}
\hline \hline $\begin{array}{c}\text { Age range } \\
(\mathrm{Myr})\end{array}$ & $\begin{array}{c}\phi_{0} \\
\left(\mathrm{pc}^{-1}\right)\end{array}$ & $\begin{array}{c}z_{\odot} \\
(\mathrm{pc})\end{array}$ & $\begin{array}{c}z_{\mathrm{h}} \\
(\mathrm{pc})\end{array}$ & $\mathrm{CC}$ \\
$(1)$ & $(2)$ & $(3)$ & $(4)$ & $(5)$ \\
\hline All ages & $5.7 \pm 0.3$ & $14.8 \pm 2.4$ & $57.2 \pm 2.8$ & 0.87 \\
$\leq 200$ & $4.0 \pm 0.3$ & $14.8 \pm 2.4^{\dagger}$ & $47.9 \pm 2.8$ & 0.93 \\
$200-1000$ & $0.5 \pm 0.1$ & $14.8 \pm 2.4^{\dagger}$ & $149.8 \pm 26.3$ & 0.64 \\
\hline
\end{tabular}

Notes: $\phi(z)=\phi_{0} \mathrm{e}^{-\left|\left(z+z_{\odot}\right) / z_{\mathrm{h}}\right|}$. Column 5: correlation coefficient.

$\dagger$ : Parameter kept fixed.

samples of different objects (stars and OCs) provide essentially the same value of the Solar displacement from the plane. For completeness we also applied the unconstrained exponentialdecay fit to the young and moderate-age OCs. For the young OCs we obtain $z_{\odot}=14.2 \pm 2.3 \mathrm{pc}$ and $z_{\mathrm{h}}=47.9 \pm 2.8 \mathrm{pc}$ which, within the uncertainties are in excellent agreement with the constrained values (Table 1). However, the unconstrained fit of the less-uniform distribution of the moderate-age OCs produced a large uncertainty in $z_{\odot}, 9.8 \pm 11.4 \mathrm{pc}$ and a similar value of $z_{\mathrm{h}}, 145 \pm 25 \mathrm{pc}$. We conclude that the value of the displacement of the Sun over the Galactic plane derived from the distribution of the young OCs is, within the uncertainties, the same as the average value which considers OCs of all ages.

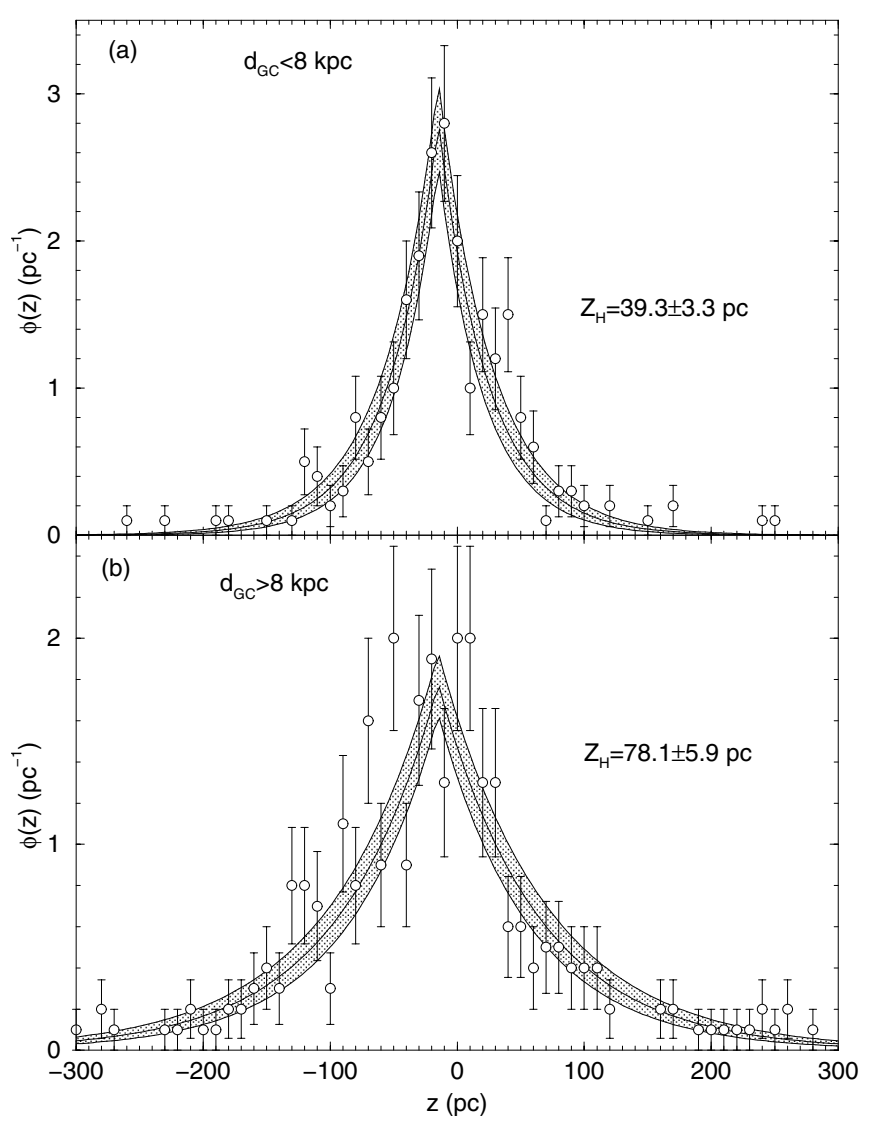

Fig. 8. $z$-distribution function of OCs (younger than $1 \mathrm{Gyr}$ ) more internal (panel a)) and more external (panel b)) than the Solar circle.

According to the above, the young OCs are distributed close to the disk with a scale height $z_{\mathrm{h}} \approx 48 \mathrm{pc}$. The scale height derived from the moderate-age OCs is $\sim 3$ times larger than that of the young OCs. Our value of $z_{\mathrm{h}}$ for the young OCs agrees with that derived by Janes \& Phelps (1994) for the similar age group. On the other hand, in the present case the old (age $\geq 1 \mathrm{Gyr}$ ) clusters are uniformly distributed in $z$, in contrast to the $z_{\mathrm{h}} \approx 375 \mathrm{pc}$ distribution derived by Janes \& Phelps (1994).

\subsection{Dependence of $\mathrm{Z}_{\mathrm{h}}$ on Galactocentric distance}

In recent years observational evidence in favour of disk thickening with increasing Galactocentric distance has been presented (e.g. Kent et al. 1991; Janes \& Phelps 1994). To examine this issue we build $z$-distribution functions for clusters more internal and more external than the Solar circle. We exclude from this analysis the old OCs because of their nearly-uniform $z$-distribution (panel d in Fig. 7). The results are in Fig. 8.

From the fits we conclude that the average scale height for disk-regions outside the Solar circle is $z_{\mathrm{h}}=78.1 \pm 5.9 \mathrm{pc}$ (correlation coefficient $\mathrm{CC}=0.87$ ), while for regions interior to the Solar circle $z_{\mathrm{h}}=39.3 \pm 3.3 \mathrm{pc}(\mathrm{CC}=0.94)$. This represents an increase in the average $z_{\mathrm{h}}$ by a factor of $\approx 2$ along the observed Galactocentric distance range. 


\section{Sample completeness simulation}

As already suggested by the histograms in Fig. 6, completeness effects are most likely affecting critically the observed radial distribution of OCs $(\phi(r))$ for large distances from the Sun, especially towards the Galactic center/bulge. The discussions in Sect. 3 pointed (i) the critical rôle played by the background stellar density in the detection of low-contrast OCs, particularly in directions intercepting the Galactic center/bulge; and (ii) the sharp decrease of observable stars above the background with increasing distance from the Sun. Consequently, the dependence of completeness on Galactocentric distance (as well as on height over the plane) has to be taken into account so that more objective results on the intrinsic spatial distribution of OCs can be drawn.

To minimize the effects of distance from the Sun and background density we restricted the following analysis to the OCs located in the shaded region shown in panel d of Fig. 1. This region contains the OCs with Galactocentric distance in the range 6.7 to $9.3 \mathrm{kpc}$ which on average are $1.3 \mathrm{kpc}$ distant from the Sun. Considering this distance along the Solar circle in both directions the restricted region is swept out by azimuthal angles in the range $-9.23^{\circ}$ to $+9.23^{\circ}$ with respect to the Galactic center. As a consequence of the resulting geometry of the region, $90 \%$ of the OCs included in the restricted sample have distances from the Sun of at most $d_{\odot}=1.3 \mathrm{kpc}$, and only $3 \%$ have $d_{\odot}$ in the range 1.5 to $1.8 \mathrm{kpc}$.

In Fig. 9 we show the distribution function $\left(\phi(r)=\frac{\mathrm{d} N_{\mathrm{oc}}}{\mathrm{d} r}\right)$ of OCs (in the restricted sample) in terms of Galactocentric distance. As already noted in Fig. 6 the observed OC distribution presents a maximum at the Sun's position and falls off both for regions internal and external to the Solar circle, with a V-shaped dip at $d_{\mathrm{GC}} \approx 7.5-7.8 \mathrm{kpc}$ (the region before the Sgr-Car Arm). van den Bergh \& McClure (1980) attributed the sharp drop in the number of OCs for regions internal to the Solar circle to enhanced OC-disruption through the frequent collisions with molecular clouds in the inner regions of the Galaxy, as well as dissolution through tidal effects due to the Galaxy. However, when compared to the expected distribution of an exponential disk, $\phi(r) \propto r \mathrm{e}^{-\left(r / R_{\mathrm{D}}\right)}$ (Sect. 7), the drop in the observed distribution for $d_{\mathrm{GC}} \leq 8 \mathrm{kpc}$ turns out exceedingly large to be accounted for by disruption related to dynamical effects alone.

Considering the above facts we derive the spatial dependence of completeness by incorporating the qualitative arguments presented in Sect. 3 to measurements of the background stellar density as seen by 2 MASS photometry in different directions around the Sun.

As a first step we measured the background stellar density of the Galaxy as a function of $\ell$ and $b$ using 2MASS photometry. We counted the number of stars brighter than $J=15$ contained in circular areas with $1^{\circ}$ in radius for the Galactic latitudes $b=0^{\circ}, \pm 2.5^{\circ}, \pm 5^{\circ}, \pm 10^{\circ}, \pm 25^{\circ}$ and $\pm 50^{\circ}$ in steps of $5^{\circ}$ in $\ell$. The selected circular area is large enough to accommodate most of the local fluctuations in the number of stars. We illustrate some of the northern background density profiles in panel a of Fig. 10, where the marked difference in the average background in directions towards the Galactic center/bulge

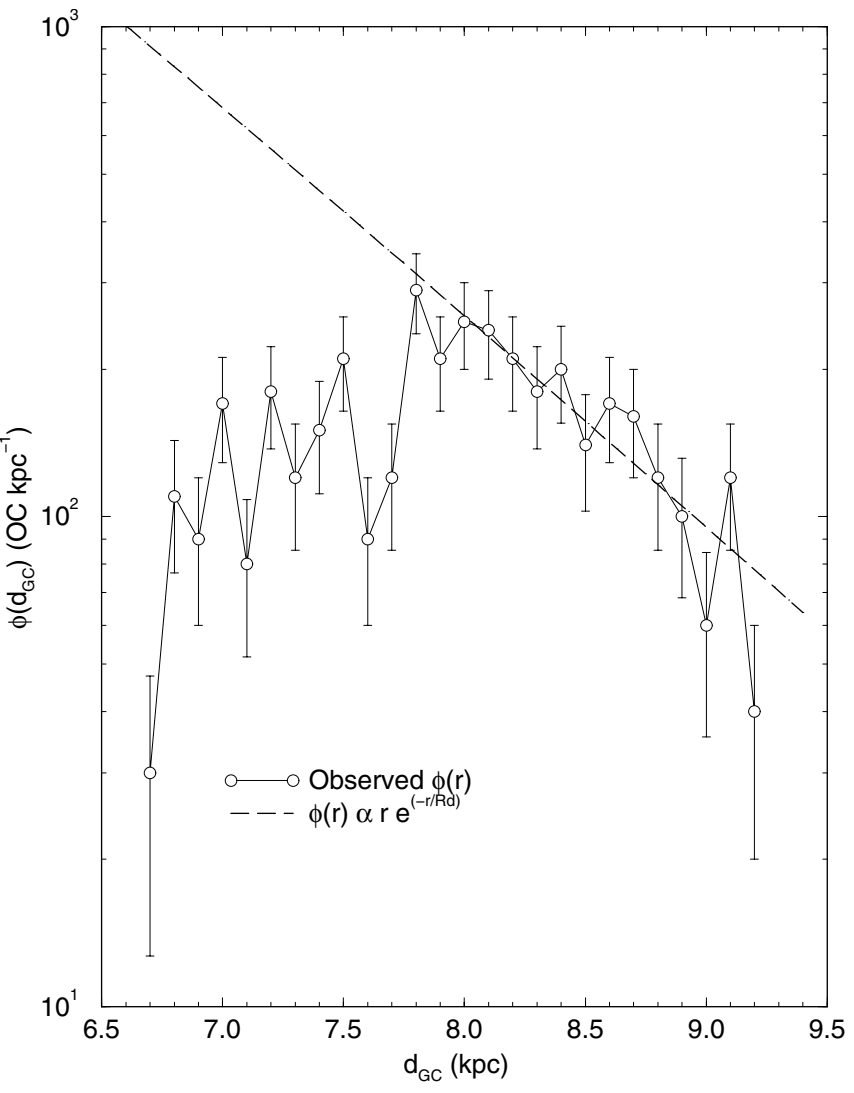

Fig. 9. Observed distribution function of $\mathrm{OCs}$ in terms of Galactocentric distance in the restricted zone. Dashed line: exponential-disk distribution $\phi(r) \propto r \mathrm{e}^{-\left(r / R_{\mathrm{D}}\right)}$. Note the depletion in the number of OCs for $d_{\mathrm{GC}} \leq 7.8 \mathrm{kpc}$, and the V-shaped dip at $d_{\mathrm{GC}} \approx 7.5-7.8 \mathrm{kpc}$.

with respect to those towards the anti-center is clear. The corresponding southern profiles are very similar to the northern ones. The background contribution for a given OC (i.e. at a specific pair of the coordinates $\ell$ and $b$ ) is obtained by interpolation among the above curves.

Our analysis is based on the detectability of OCs - particularly the more distant ones - projected towards different regions of the Galaxy, a process which depends essentially on the density contrast of the central region of a cluster with respect to the field. In this sense we assume a typical angular radius of $1^{\prime}$ to characterize the central region of the clusters. To estimate the effective number of stars of the OCs in the restricted zone we counted the number of stars brighter than $J=15(\sim \sigma(0))$ within the $R=1^{\prime}$ central region, regardless of the OC distance from the Sun. The corresponding background contribution is subtracted therefrom to yield the effective number of stars, $N *=\sigma(0)-\sigma_{\mathrm{bg}}=\sigma_{0 \mathrm{~K}}$.

To avoid the high background density towards the Galactic center/bulge we restricted this procedure to the OCs external to the Solar circle. We assumed the WEBDA coordinates as the center of the OCs from which we extracted the photometry. The resulting histogram of the number of OCs with a given effective number of stars is shown in panel b of Fig. 10 (solid line). For a more objective comparison of the effective stellar content among the OCs we recalculated $N *$ assuming 

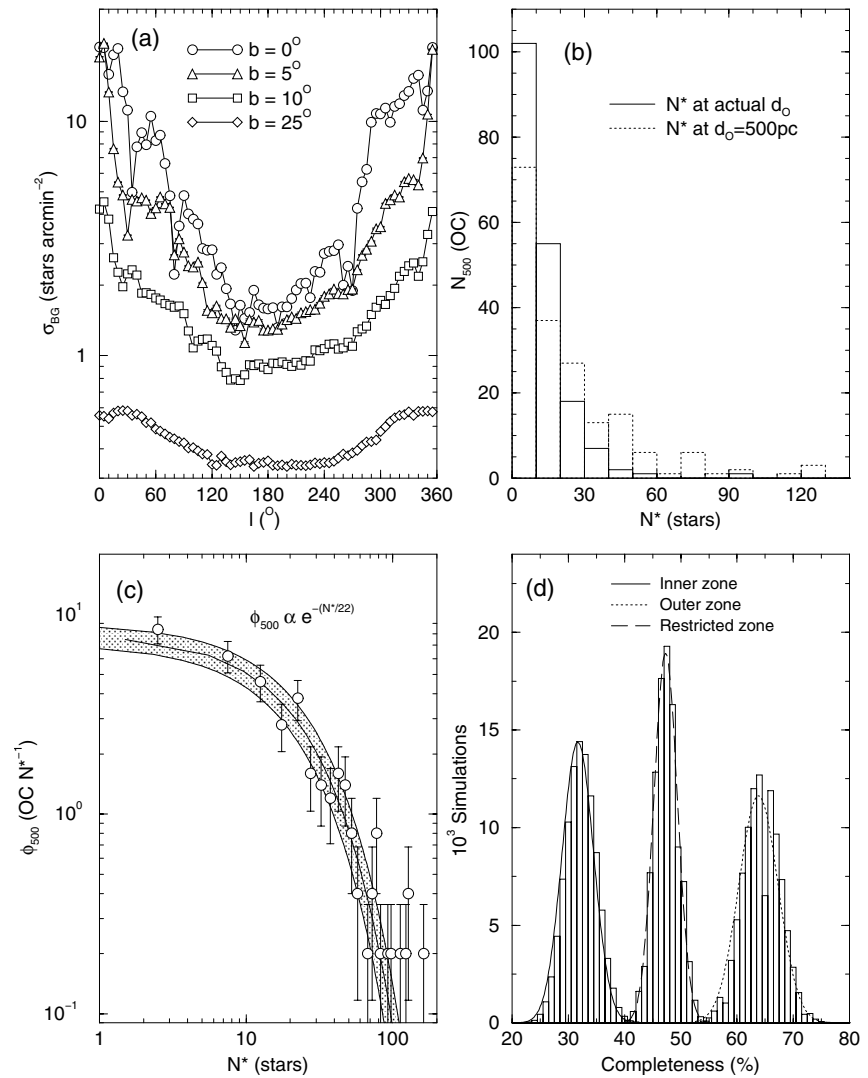

Fig. 10. Panel a): average background density (2MASS stars brighter than $J=15$ ) to the north of the Galactic plane. $1 \sigma$-Poisson errors are smaller than the symbols. Panel b): histograms of the number of OCs (outside the Solar circle in the restricted zone) with a given effective number of stars at the actual distance from the Sun (solid line) and at $d_{\odot}=500 \mathrm{pc}$ (dotted line). Panel c): the distribution function at $d_{\odot}=$ $500 \mathrm{pc}$ can be fitted by $\phi_{500}(N *) \propto \mathrm{e}^{-(N * / 22)}$; Shaded area: $1 \sigma$ standard deviation from the fit. Panel d): average integrated completeness for the OCs inside (solid line) and outside (dotted line) the Solar circle and throughout the restricted zone (dashed line).

$d_{\odot}=500$ pc for all OCs, using the relation $N *\left(d_{\odot}\right) \sim d_{\odot}{ }^{-1}$ (Sect. 3). The resulting histogram is in panel b of Fig. 10 (dotted line). Interestingly, the corresponding distribution function (panel c) is well represented by an exponential-decay profile, $\phi_{500}(N *) \propto \mathrm{e}^{-(N * / 22)}$.

OCs with off-center coordinates in WEBDA and those with large projected areas such as the Hyades and Pleiades would simply enhance the low- $N *$ tail of the $\phi_{500}(N *)$ distribution (panel c of Fig. 10), which would not change appreciably the shape of the distribution.

Our basic assumption is that the observed distribution of the number of OCs with a given effective number of stars outside the Solar circle $\left(\phi_{500}(N *)\right)$ both represents the intrinsic distribution of OCs in the Galaxy and is essentially isotropic with respect to the Sun. Based on this we randomly assign to each observed OC position $\left(\ell, b, d_{\odot}\right)$ in the restricted zone a value of $N *$ (corresponding to $d_{\odot}=500 \mathrm{pc}$ ) with a numberfrequency distributed according to the observed histogram (panel b of Fig. 10), and recalculate $N *$ for the actual distance from the Sun of the OC. Finally, we divide this last number by the corresponding number of background stars (calculated at

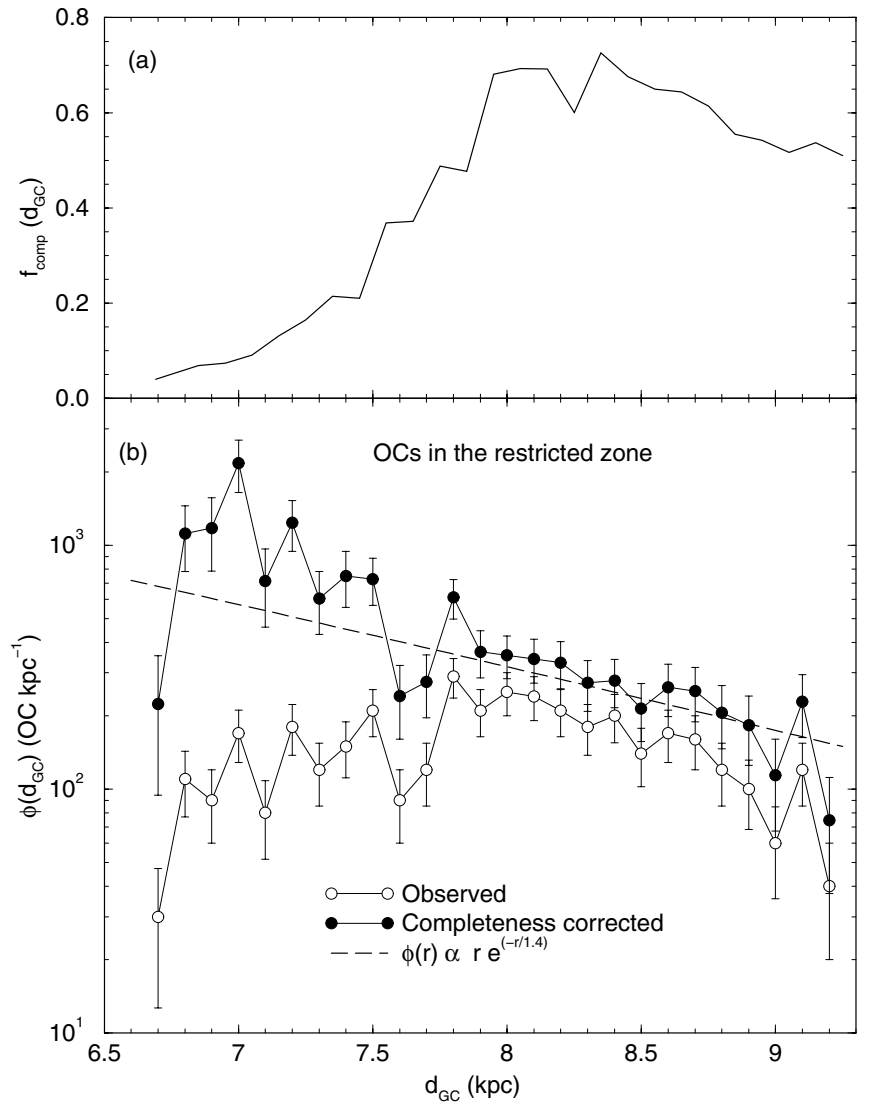

Fig. 11. Panel a): completeness fraction as a function of Galactocentric distance in the restricted zone. Panel b): observed (empty circles) and completeness-corrected (filled circles) OC distributions. Dashed-line: fit with an exponential-disk with a scale-length $R_{\mathrm{D}}=1.4 \pm 0.2 \mathrm{kpc}$.

the respective $\ell, b)$ to obtain the contrast parameter $\delta_{\mathrm{c}}=$ $1+\frac{N *}{\sigma_{\mathrm{bg}}}$. We count as detectable only the OCs with $\delta_{\mathrm{c}} \geq 2$ (i.e. $\left.\frac{N *}{\sigma_{\mathrm{bg}}} \geq 1\right)$. Completeness at a given position is then the fraction of retrieved OCs with respect to the input ones. To test convergence of this procedure we ran $10^{5}$ simulations. We illustrate the convergence of this process in panel d of Fig. 10 where we plot histograms of the resulting integrated completeness value with respect to the number of simulations, both for the OCs inside and outside the Solar circle. The histograms are well represented by gaussian distributions centered at $31.7 \%$ and $63.8 \%$, with standard deviations of $2.8 \%$ and $3.5 \%$, respectively for the OCs inside and outside the Solar circle. As expected because of the higher background density, the average completeness for the OCs inside the Solar circle is about half of that for the OCs outside it. The average integrated completeness throughout the entire restricted zone is $47.3 \pm 2.1 \%$.

The completeness fraction as a function of Galactocentric distance (for the OCs in the restricted zone) is shown in panel a of Fig. 11. As expected, the completeness is highest around the Sun $\left(f_{\text {comp }}(8 \mathrm{kpc}) \approx 0.68\right)$ and drops off towards external regions and more sharply towards the Galactic center. Dividing the observed OC distribution function (Fig. 9) by $f_{\text {comp }}(r)$

$$
3 \frac{N *}{\sigma_{\mathrm{bg}}}=\frac{\sigma_{0 \mathrm{~K}}}{\sigma_{\mathrm{bg}}}=\delta_{\mathrm{c}}-1 .
$$


produces a completeness-corrected radial distribution which follows the expected exponential-disk profile (panel b of Fig. 11) along the entire Galactocentric distance range of the restricted zone, with a scale-length $R_{\mathrm{D}}=1.4 \pm 0.2 \mathrm{kpc}$. This estimate of $R_{\mathrm{D}}$ represents about $40 \%$ of the stellar overall disk scale-length (de Vaucouleurs \& Pence 1978). Our determination probably reflects the Solar-vicinity value of $R_{\mathrm{D}}$ and/or that the OCs distribute in the disk with a different scale length than the stars. Remark that the V-shaped dip at $d_{\mathrm{GC}} \approx 7.5-7.8 \mathrm{kpc}$ (attributed to the edge before the Sgr-Car Arm - Sect. 2.1) is still present in the completeness-corrected distribution function. In this sense, the above completeness-simulation procedure is sensitive as well to conspicuous physical structures of the Galaxy.

Since our analysis is based on magnitude-limited 2MASS photometry $(J=15)$, excess reddening of background sources towards the inner Galaxy with respect to those in the anticenter (Fig. 5) may have underestimated the contrast parameter of the OCs in that region because of the increased differential brightness of the brightest stars. As a consequence, completeness for the OCs in the inner Galaxy would be underestimated, which might account for the over-correction of $\phi\left(d_{\mathrm{GC}}\right)$ with respect to the exponential-disk fit, as suggested by Fig. 11 for $d_{\mathrm{GC}} \leq 7.5 \mathrm{kpc}$.

Considering the completeness-corrected OC distribution, the fraction of observed OCs in the restricted zone corresponds to about $47 \%$ of the probable total (essentially of Trumpler types I to III - Sect. 3).

\section{Extension to the whole OC sample}

In the previous section we corrected for completeness the observed radial distribution of OCs in the restricted zone and, as a result obtained the expected exponential-disk profile. At this point it is interesting to check whether the completeness-simulation procedure retrieves an exponentialdisk profile when applied to the whole OC sample. We test this hypothesis considering 2 different simulation approaches.

\subsection{Actual-position simulation}

In this simulation the effective number of stars (Sect. 5) $N *$ is randomly distributed (with the number-frequency according to the histogram in panel b of Fig. 10) among the 654 OCs assuming the WEBDA spatial locations $\left(\ell, b, d_{\odot}\right)$. The completeness fraction of each $O C$ is then the average value of the retrieval rate after running $10^{5}$ simulations. Similarly to the restrictedzone simulation, the individual $1 \sigma$-standard deviation of the completeness fraction is at most $3 \%$ of the corresponding average value.

The observed radial distribution of OCs in the range $5 \leq$ $d_{\mathrm{GC}}(\mathrm{kpc}) \leq 14$ (Fig. 12) presents a maximum at the Solar circle and drops off both towards larger Galactocentric distances and more sharply to the Galactic center. The radial completeness fraction resulting from the actual-position simulation (Fig. 13) presents similar features. Correction of the observed distribution for completeness produces a radial distribution which follows an exponential-disk profile (panel a) characterized by

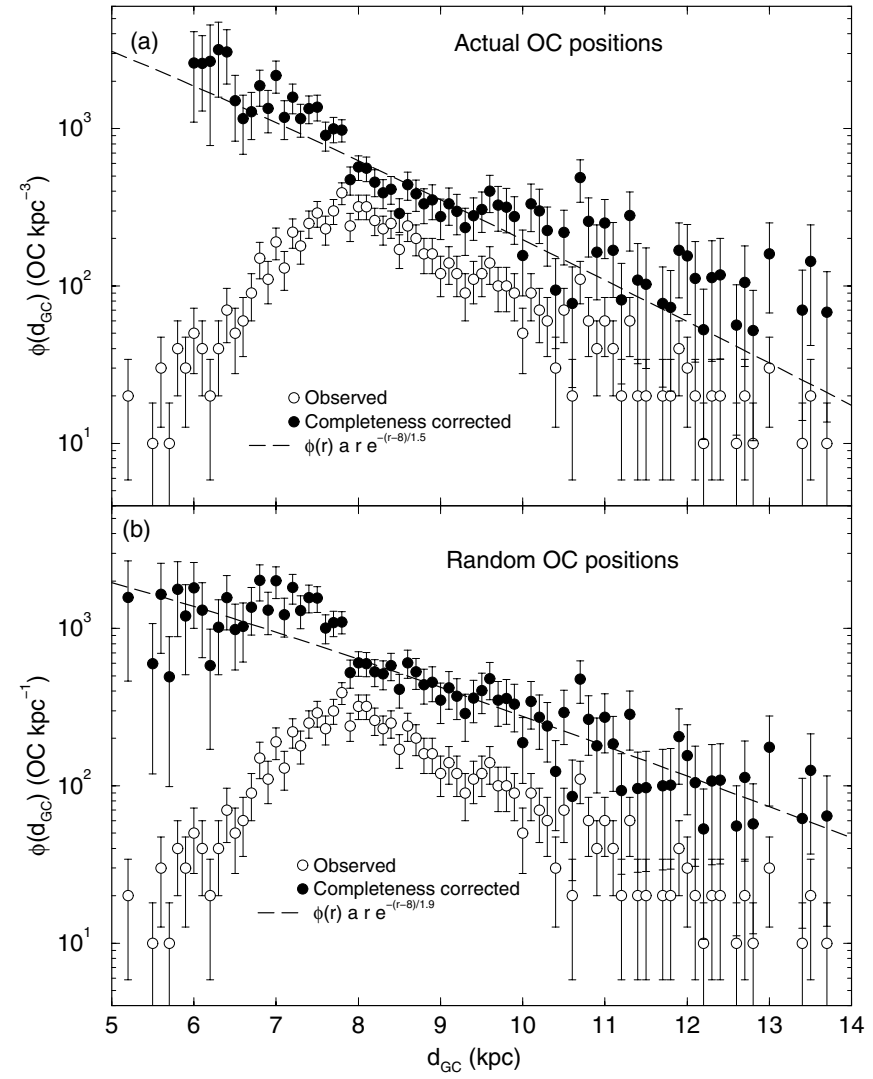

Fig. 12. Completeness-correction of the observed radial distribution of OCs (empty circles) according to the actual-position (top panel) and random-position (bottom panel) simulations. The resulting radial distributions (filled circles) follow exponential-disk profiles characterized by scale-lengths $R_{\mathrm{D}} \approx 1.5 \mathrm{kpc}$ and $R_{\mathrm{D}} \approx 1.9 \mathrm{kpc}$, respectively.

a scale-length of $R_{\mathrm{D}}=1.5 \pm 0.1 \mathrm{kpc}$ (correlation coefficient $\mathrm{CC}=0.88)$.

The average integrated completeness throughout the disk is $33.2 \pm 1.5 \%$.

\subsection{Random-position simulation}

In this case we build 1000 artificial test positions $\left(\ell, b, d_{\odot}\right)$ over which we randomly distribute the effective number of stars as in the actual-position simulation. The $\ell$ coordinate is randomly selected around the Sun while $b$ is drawn according to the observed number-frequency histogram shown in panel e of Fig. 3; to match most of the observed distances from the Sun (panel a of Fig. 4), $d_{\odot}$ is randomly selected from the range $0-7 \mathrm{kpc}$. Because of the random nature of the test OC positions, the resulting radial completeness fraction (Fig. 13) turns out similar to, but considerably smoother than that derived in the actualposition simulation.

The completeness-corrected radial distribution of OCs (panel b of Fig. 12) follows an exponential-disk profile characterized by a scale-length $R_{\mathrm{D}}=1.9 \pm 0.1 \mathrm{kpc}(\mathrm{cc}=0.88)$, about $30 \%$ larger than that obtained with the actual-position simulation, within the uncertainties. 


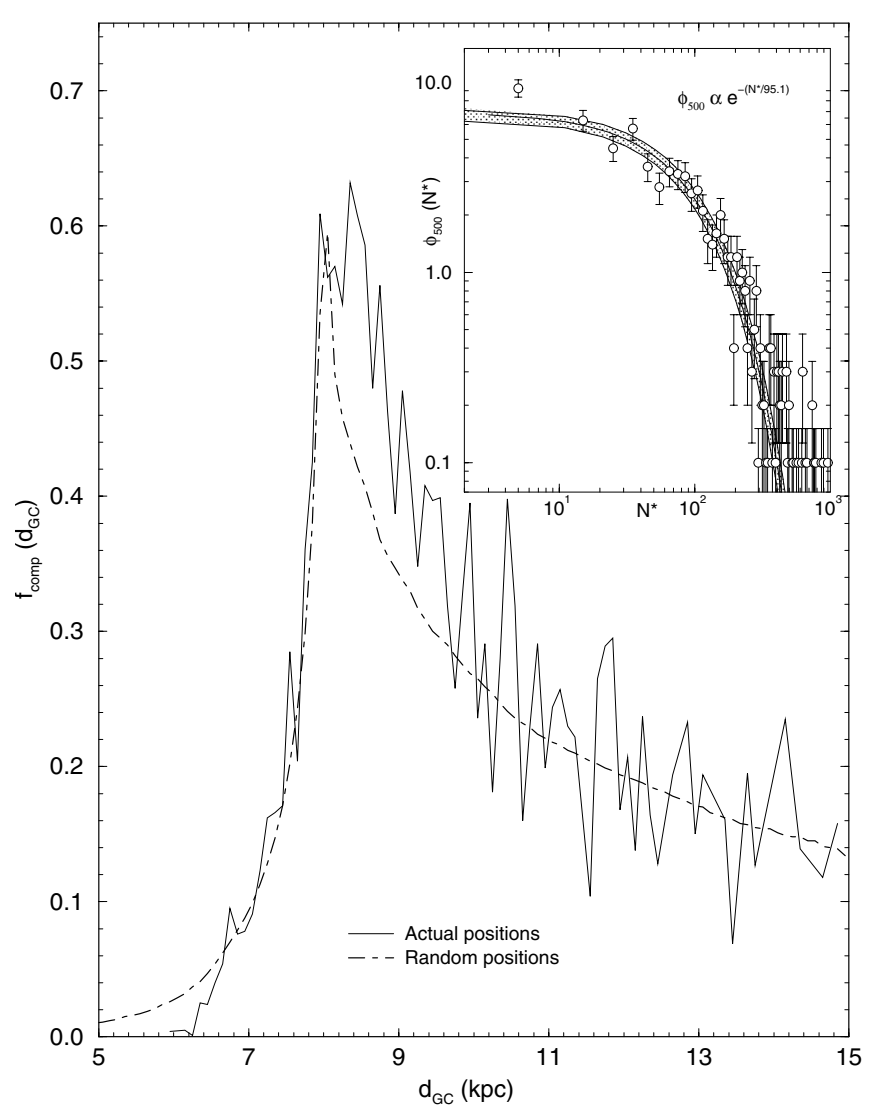

Fig. 13. The actual-position radial completeness fraction (solid line) drops off more sharply towards the Galactic center for $d_{\mathrm{GC}} \leq$ $6.6 \mathrm{kpc}$ than that of the random-position simulation (dot-dashed line). Inset: the distribution of the number of OCs with a given effective (background-subtracted) number of stars for the whole OC sample is well fitted by an exponential-decay profile. Shaded area: $1 \sigma$ standard deviation from the fit.

\subsection{Comparison of simulations}

The simulations discussed above are based on conceptually different approaches with respect to the spatial distribution of OCs. Despite this, they produced similar completenesscorrected radial distributions of OCs, which enhances the significant rôle played by completeness in depth-limited photometric surveys throughout the Galaxy. However, the completeness-corrected profile obtained with the randomposition simulation produces a visually better overall fit with the expected exponential-disk profile (Fig. 12) as compared to the actual-position simulation, especially near the inner and outer $d_{\mathrm{GC}}$ borders. The completeness-corrected distributions derived from both simulation approaches present similar disk scale lengths, $R_{\mathrm{D}} \approx 1.5-1.9 \mathrm{kpc}$, about $43 \%-54 \%$ of the canonical value $\left(R_{\mathrm{D}} \approx 3.5 \mathrm{kpc}\right)$ derived with stars by de Vaucouleurs $\&$ Pence (1978). This fact may reflect intrinsic differences in the spatial distribution of OCs with respect to stars.

In Fig. 13 we compare the radial completeness fractions resulting from both simulations. They basically agree, except for $d_{\mathrm{GC}} \leq 6.6 \mathrm{kpc}$ where the actual-position completeness fraction drops off more sharply towards the Galactic center than that from the random-position simulation. Considering that in the random-position simulation the OCs are uniformly distributed throughout the Galaxy, as opposed to observed OC coordinates in the actual-position, perhaps this difference may suggest that the tidal-disruption effects due to the Galactic center/bulge begin to be observationally significant at $d_{\mathrm{GC}} \approx 6.6 \mathrm{kpc}$.

Finally, in the inset of Fig. 13 we show the distribution of the number of OCs with a given effective (backgroundsubtracted) number of stars for the whole OC sample. Similarly to the OCs in the restricted zone this distribution follows an exponential-decay profile, but with a characteristic number of stars of $\Delta N * \approx 95$. The decay-scale for the whole sample is about 4 times larger than that derived for the OCs outside the Solar circle in the restricted zone (Sect. 5). This discrepancy can be accounted for by an observational bias, since detection probability in directions intercepting the Galactic center/bulge is higher for the more populous OCs. Interestingly, the analytical expression of the overall $\phi_{500}$ resembles that of the galaxy luminosity function (Schechter 1976).

\subsection{Completeness in vertical distributions}

In Sect. 4.1 we showed that the observed scale height depends on Galactocentric distance, in the sense that the OC distribution outside the Solar circle is about $2 \times$ broader than that inside it. At this point it may be interesting to determine how completeness affects the vertical distribution and derive its intrinsic shape.

In Fig. 14 (left panel) we show how the completeness fraction as a function of $\mathrm{z}$ depends on Galactocentric distance, centered at the Solar position over the disk $\left(z_{\odot}=14.81 \mathrm{pc}\right.$, Sect. 4$)$. The curves have been produced with the random-position simulation (Sect. 6.2). As expected, the OCs internal to the Solar circle suffer more severely from completeness than the external ones. Interestingly, both curves present similar shapes, with a peak at $z=z_{\odot}$, sharp declines for increasing $|z|$ with a minimum at $|z| \approx 340 \mathrm{pc}$, and an increase for larger values of $|z|$. As shown in the right panel OCs at different ranges of distance from the Sun present similar dependence with $z$, with a minimum at $z=z_{\odot}$ and an increase for larger $|z|$. However, the absolute values of completeness drop for more distant OCs. The final shape of the completeness fraction results from the combination of OCs at different distances from the Sun. In particular, the peak at $z=z_{\odot}$ can be accounted for by the high completeness of the OCs closer than $\approx 1 \mathrm{kpc}$ from the Sun.

The observed $z$-distributions of OCs internal and external to the Solar circle, as well as the overall one have been corrected for completeness with the corresponding curves derived above. The resulting completeness-corrected distributions along with the respective exponential-decay fits are shown in Fig. 15. The completeness-corrected scale heights are $z_{\mathrm{h}}=78 \pm 3 \mathrm{pc}$ (observed: $z_{\mathrm{h}} \approx 57 \mathrm{pc}$ ) with correlation coefficient $\mathrm{CC}=0.88$, $z_{\mathrm{h}}=85 \pm 13 \mathrm{pc}$ (observed: $z_{\mathrm{h}} \approx 39 \mathrm{pc}$ ) with $\mathrm{CC}=0.77$, and $z_{\mathrm{h}}=119 \pm 12 \mathrm{pc}$ (observed: $z_{\mathrm{h}} \approx 78 \mathrm{pc}$ ) with $\mathrm{CC}=0.75$, respectively for the overall, internal and external to the Solar circle distributions. Consequently, completeness correction of the $z$-distributions preserved the dependence of scale height on Galactocentric distance, increased the absolute values of $z_{\mathrm{h}}$ 


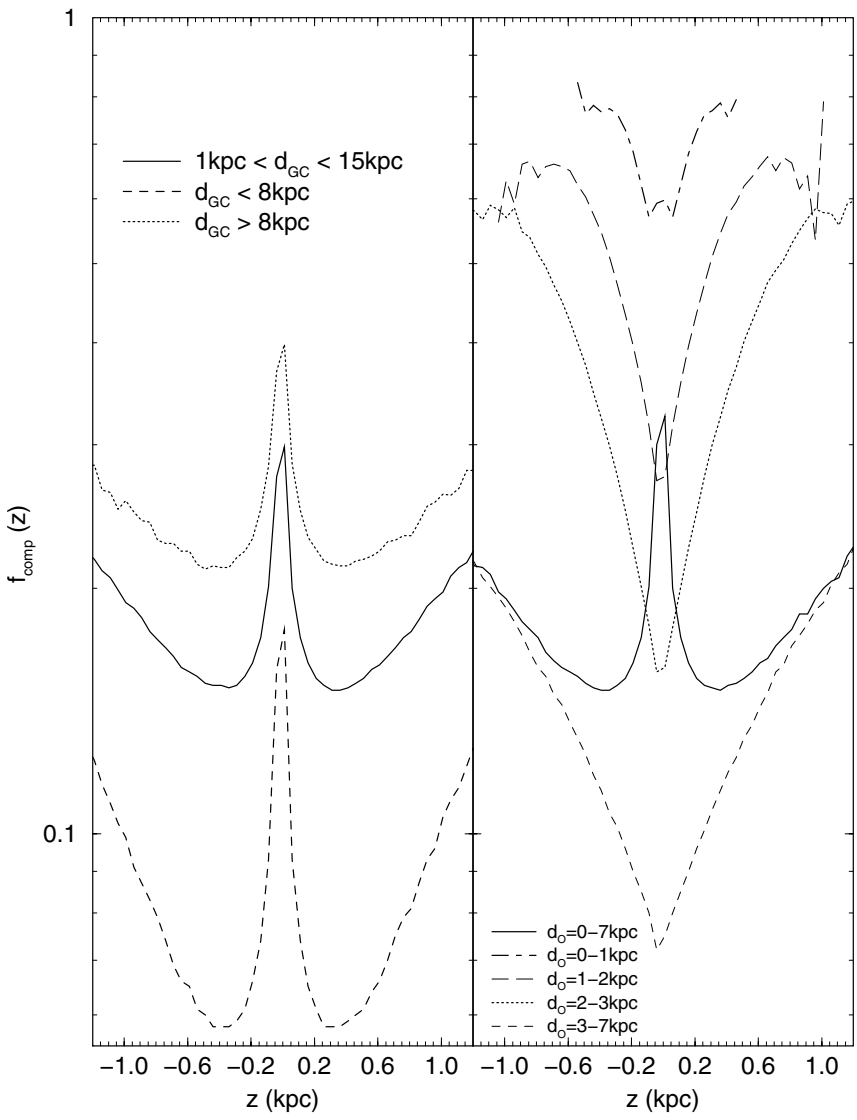

Fig. 14. Completeness fraction as a function of $z$. Left panel: completeness fraction for OCs internal (dashed line) and external (dotted line) to the Solar circle, and the average disk completeness (solid line). Right panel: the shape of the average disk completeness results from the combination of OCs at different ranges of distance from the Sun.

with respect to the observed ones and decreased the observed ratio of $z_{\mathrm{h}}$ outside/inside the Solar circle from 2 to 1.4.

Completeness-correction of the observed $z$-distributions of the OCs younger than $200 \mathrm{Myr}$ and those with age in the range $200 \mathrm{Myr}-1 \mathrm{Gyr}$ (panels $\mathrm{b}$ and $\mathrm{d}$ of Fig. 7, respectively) increased the scale height by a factor of $\sim 1.42$. Consequently, the young OCs distribute vertically following an exponential-decay profile with scale height $z_{\mathrm{h}} \approx 68 \mathrm{pc}$. For the $200 \mathrm{Myr}-1 \mathrm{Gyr}$ OCs the completeness-corrected scale height is $z_{\mathrm{h}} \approx 230 \mathrm{pc}$. Considering the observational uncertainties, these values are comparable to the H I scale height in the Solar neighbourhood, which we take as $\sim \frac{1}{2} F W H M(\mathrm{HI}) \approx 115 \mathrm{pc}$ (Dickey \& Lockman 1990). This must reflect the association of star formation and the parent gas. The same conclusion applies to $\mathrm{CO}$, for which surveys of the outer Galaxy (Heyer et al. 1998), where the Perseus arm lies, find an overall mean CO scale height of $113 \mathrm{pc}$.

\subsection{Age distribution function}

The histogram with the number of observed OCs in bins of age (Fig. 2) was converted into the observed age-distribution function (panel a of Fig. 16). This function can be fitted by a combination of exponential-decay profiles with age scales of

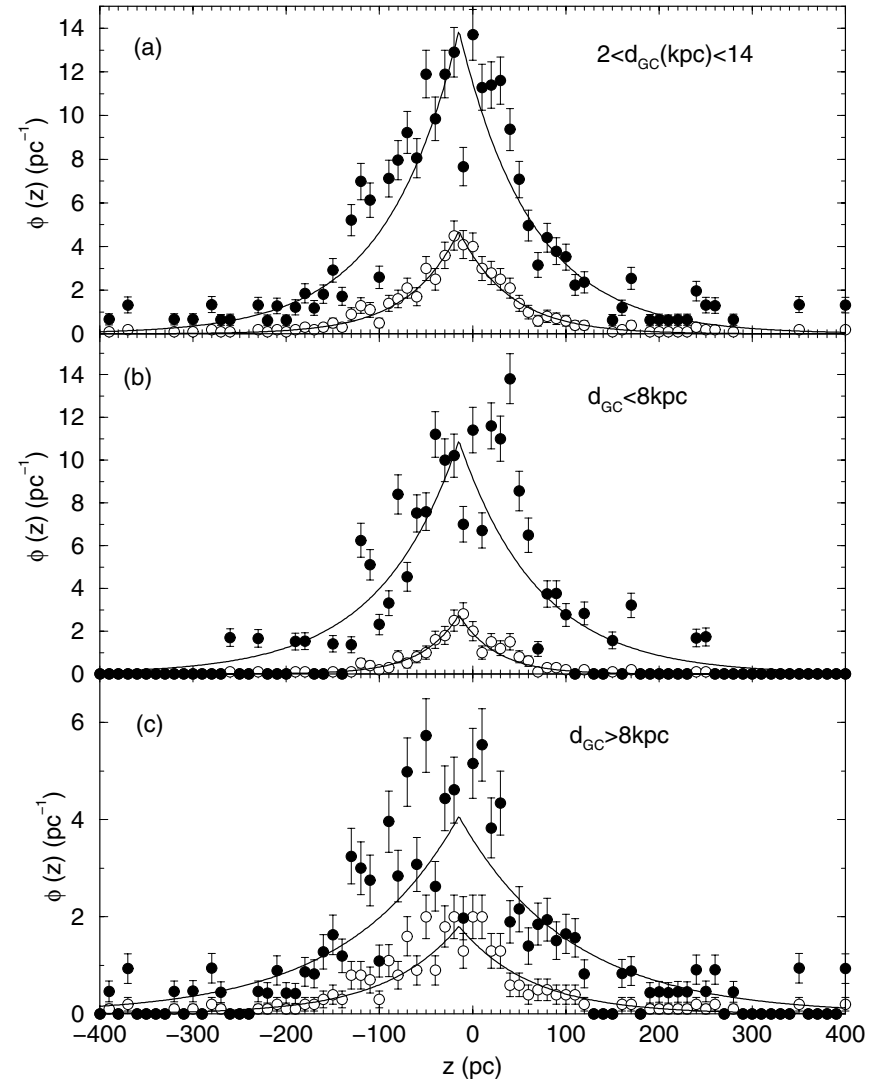

Fig. 15. Completeness-corrected $z$-distributions. Panel a): overall radial distribution (scale height $z_{\mathrm{h}} \approx 78 \mathrm{pc}$ ). Panel b): OCs internal to the Solar circle $\left(z_{\mathrm{h}} \approx 85 \mathrm{pc}\right)$. Panel c): OCs external to the Solar circle $\left(z_{\mathrm{h}} \approx 119 \mathrm{pc}\right)$. Empty circles: observed OCs. Filled circles: completeness-corrected $z$-distribution.

$\tau_{\text {young }}=123 \pm 13 \mathrm{Myr}$ (characteristic of the young OC population) and $\tau_{\text {old }}=2.4 \pm 1 \mathrm{Gyr}$ (old OCs). The correlation coefficient of the fit is $\mathrm{CC}=0.94$. Clearly the observed old OC population represents an excess over the extrapolation to old ages of the young OCs distribution function. An additional excess in the observed age-distribution function shows up in the age range $\sim 600 \mathrm{Myr}$ to $\sim 1.5 \mathrm{Gyr}$, which can probably be identified with the moderate-age OC population. The present young and old age time-scales are comparable to those derived by Janes \& Phelps (1994), $200 \mathrm{Myr}$ and $4 \mathrm{Gyr}$ for the young and old OCs, respectively.

The observed age-distribution function (panel a of Fig. 16) basically contains the survivor clusters after emerging from the parent molecular clouds. Lada \& Lada (2003) estimate that, for embedded clusters within $2 \mathrm{kpc}$ from the Sun, only a fraction of 4-7\% emerge as OCs. Bica et al. (2003a) compiled 276 embedded clusters (mostly infrared clusters) and stellar groups from the literature located mostly within $3 \mathrm{kpc}$ from the Sun. From the age-distribution function in Fig. 16 we estimate that the number of observed OCs with ages in the ranges 5-10 Myr, 10$15 \mathrm{Myr}$, and $15-20 \mathrm{Myr}$ are $21 \pm 3,21 \pm 3$ and $20 \pm 3$, respectively. Considering an age range of $5 \mathrm{Myr}$ for the embedded clusters (typical lifetime of H II regions) and comparing with the number of OCs in the subsequent 5 Myr-bins (see above) we can derive a survival rate of $\sim 8 \%$, similar to Lada \& Lada (2003)'s 


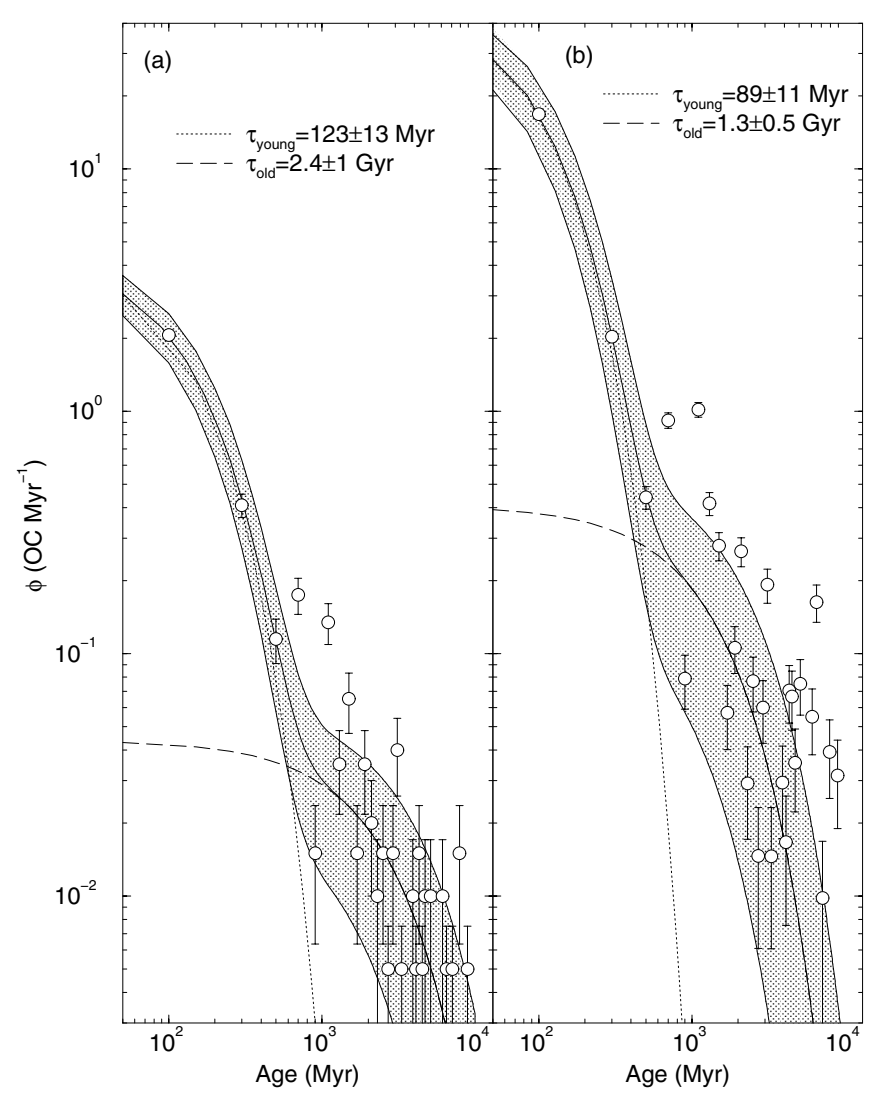

Fig. 16. Panel a): the observed age-distribution can be fitted with two exponential-decay laws with time-scales of $\tau_{\text {young }}=123 \pm 13 \mathrm{Myr}$ (dotted line) and $\tau_{\text {old }}=2.4 \pm 1 \mathrm{Gyr}$ (dashed line) characterizing the young and old OCs, respectively. Panel b): same as a) for the completenesscorrected age distribution; time-scales are $\tau_{\text {young }}=89 \pm 11$ Myr (dotted line) and $\tau_{\text {old }}=1.3 \pm 0.5 \mathrm{Gyr}$ (dashed line). Shaded region: $1 \sigma$-standard deviation of the combined young+old fit.

upper limit. More recently Dutra et al. (2003) and Bica et al. (2003b) surveyed for new embedded clusters, groups and candidates in the direction of radio and optical H II regions and molecular clouds. These objects are typically within $\sim 5 \mathrm{kpc}$ from the Sun according to kinematical distances of the related $\mathrm{H}$ II regions. Adding these newly discovered embedded clusters to those of Bica et al. (2003a), totaling 622 objects, the survival rate drops to $\approx 3.4 \%$. The present estimates match those of Lada \& Lada (2003).

One output of the actual-position simulation (Sect. 6.1) is the average individual completeness of each OC in the present sample. The reciprocal of the completeness is essentially the detection probability of that particular OC - and consequently of its intrinsic parameters, such as the age. In this sense we can apply the same procedure to correct the observed age distribution for completeness. The resulting completeness-corrected age distribution (panel $b$ of Fig. 16) retains the basic features of the observed one, including the intermediate-age OC excess. However, because the distribution in Galactic latitude of the young OCs is narrower than that of the old OCs (Fig. 3), the young OCs are subject on average to higher incompleteness. Consequently, completeness correction should enhance the number of young OCs relative to the old ones. Indeed, the observed ratio of the number of OCs younger than $1 \mathrm{Gyr}$ with respect to the older ones is $\approx 7$, while in the completenesscorrected distribution this value increases to $\approx 14$.

Similarly to the observed profile, the best-fit of the completeness-corrected age distribution results from a combination of exponential-decay curves with time scales $\tau_{\text {young }}=$ $89 \pm 11 \mathrm{Myr}$ and $\tau_{\text {old }}=1.3 \pm 0.5 \mathrm{Gyr}(\mathrm{CC}=0.94)$, respectively for the young and old OCs. The completeness-corrected time scales result comparable to the observed ones, within the uncertainties. We note however that the resulting time scales of both OC populations correspond to about half of the corresponding values estimated by Janes \& Phelps (1994). This might suggest faster destruction-rates than those implied by Janes \& Phelps (1994), and particularly with respect to the $\sim 600 \mathrm{Myr}$ destruction-time scale suggested by Bergond et al. (2001).

From the above we conclude that evolutionary scenarios based on constant rates of cluster formation and disruption do not apply to the double exponential-decay age distribution. Probably a time-varying star-formation rate, such as a massive initial burst at the Gyr scale, might fit the data. In this context, the suggestion of a galaxy merger in the early phases of the Milky Way (Chen et al. 2001) and the subsequent disturbances to the disk and burst of star formation, could account for the observed excess in the number of old OCs surviving to the present time. One clue to disentangle the cluster formation/disruptionrate problem may be provided by the systematic study of OC remnants (e.g. Pavani et al. 2003).

\subsection{Galactocentric distance vs. age}

In Fig. 17 we examine the relation of Galactocentric distance with age. We divided the OC sample in bins of age and calculated the average age and $d_{\mathrm{GC}}$ in each bin, as well as the respective standard deviations. Lyngå (1982) and Tadross et al. (2002) concluded that older clusters are found preferentially in the outer parts of the Galaxy, while younger clusters are more evenly distributed. The observed OC distribution is in panel a and the completeness-corrected one is in panel b. The large $1 \sigma$-standard deviation bars in the observed distribution, particularly in $d_{\mathrm{GC}}$, preclude any definitive conclusion on this issue.

However there appears to exist a trend in which older clusters are found preferentially at larger Galactocentric distances. This trend is enhanced after applying the completeness correction to the observed distribution (panel b). A linear-least squares fit to the completeness-corrected distribution results in the relation $\left\langle d_{\mathrm{GC}}\right\rangle=(7.83 \pm 0.27)+(0.0012 \pm 0.0003)\langle$ Age $\rangle$ $(\mathrm{CC}=0.87)$, for $d_{\mathrm{GC}}$ in $\mathrm{kpc}$ and Age in Myr.

\section{Estimate of the total number of open clusters}

We describe the spatial number-density of open clusters in the disk as a combination of exponential-decay laws for the radial and vertical components, $\rho(r, z)=\frac{\mathrm{d} N_{\mathrm{oc}}}{r \mathrm{~d} d z \mathrm{~d} \theta}=\rho_{\mathrm{o}} \mathrm{e}^{-\left(r / R_{\mathrm{D}}\right)} \mathrm{e}^{-\left|z / z_{\mathrm{b}}\right|}$, where $R_{\mathrm{D}}$ is the disk scale-length, $z_{\mathrm{h}}$ is the scale-height and $\rho_{\mathrm{o}}$ is the number-density of OCs at the Galactic center. Using cylindrical coordinates and defining $D_{\odot}$ as the Galactocentric distance of the Sun, $z_{\odot}$ the displacement of the Sun from the Galactic plane, and $\rho_{\odot}$ the local (solar vicinity) number-density 


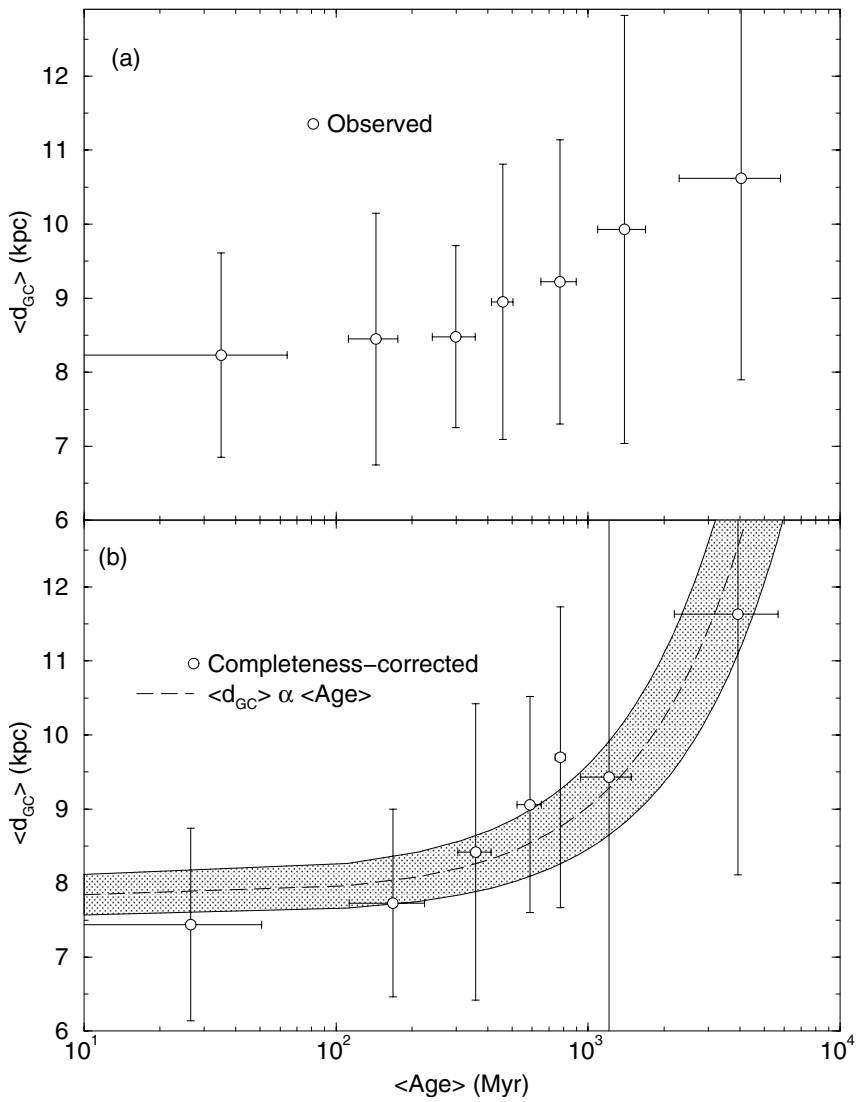

Fig. 17. Relation of Galactocentric distance and age. Panel a): observed OCs. Panel b): completeness-corrected. The bars show the $1 \sigma-$ standard deviations of the average values of age and Galactocentric distance within the respective bins. Dashed line: linear-least squares fit to the completeness-corrected points. Shaded region: $1 \sigma$-standard deviation of the fit.

of OCs, we can write $\rho(r, z)=\rho_{\odot} \mathrm{e}^{-\left(\frac{r-D_{\odot}}{R_{\mathrm{D}}}\right)} \mathrm{e}^{-\left(\frac{\left(t-z_{\odot}\right.}{z_{\mathrm{h}}}\right)}$. Integration over the $z$-axis and through a region with opening angle $\Delta \theta$ produces the radial distribution function of OCs

$\phi(r)=\frac{\mathrm{d} N_{\mathrm{oc}}}{\mathrm{d} r}=4 \pi\left(\frac{\Delta \theta}{2 \pi}\right) \rho_{\odot} r z_{\mathrm{h}} \mathrm{e}^{\frac{z \odot}{\mathrm{h}_{\mathrm{h}}}} \mathrm{e}^{-\left(\frac{r-D_{\odot}}{R_{\mathrm{D}}}\right)}$.

The total number of open clusters in the disk is then $(\Delta \theta=2 \pi)$

$N_{\text {oc }}=4 \pi \rho_{\odot} z_{\mathrm{h}} \mathrm{e}^{\frac{z_{\odot}}{z_{\mathrm{h}}}} \mathrm{e}^{\left(\frac{D_{\odot}}{R_{\mathrm{D}}}\right)} R_{\mathrm{D}}^{2}\left[1-\left(1+\frac{R_{\mathrm{G}}}{R_{\mathrm{D}}}\right) \mathrm{e}^{-\left(\frac{R_{\mathrm{G}}}{R_{\mathrm{D}}}\right)}\right]$,

where $R_{\mathrm{G}}$ is the disk radius $\left(R_{\mathrm{G}} \approx 15 \mathrm{kpc}\right.$, Binney \& Tremaine 1987$)^{4}$. Accordingly, estimates of both the solarvicinity OC density and the local disk scale length can be obtained by fitting Eq. (2) to completeness-corrected distribution functions.

Based on Eq. (2) and the completeness-corrected distribution functions of the actual-position and random-position simulations for the whole OC sample (with $\frac{\Delta \theta}{2 \pi} \approx 0.11$, Fig. 1) we derive $\rho_{\odot}=759 \pm 34 \mathrm{kpc}^{-3}$ and $\rho_{\odot}=774 \pm 33 \mathrm{kpc}^{-3}$, respectively (Fig. 12).

\footnotetext{
${ }^{4}$ Because of the exponential factor in Eq. (3) $\mathrm{e}^{-\left(\frac{R_{\mathrm{G}}}{R_{\mathrm{D}}}\right)}$ with $R_{\mathrm{D}}=$ $1.5-1.9 \mathrm{kpc}$ (Sects. 6.1 and 6.2), our estimates of $N_{\text {oc }}$ are essentially insensitive to values of $R_{\mathrm{G}} \geq 10 \mathrm{kpc}$.
}

Extrapolation of the above values of $\rho_{\odot}$ and $R_{\mathrm{D}}$ to the whole disk using Eq. (3) produces a total number of OCs in the Galaxy of $\sim 3.7 \times 10^{5}$ and $\sim 1.8 \times 10^{5}$, respectively for the actual-position and random-position completeness-corrected distribution functions. These estimates were obtained by direct extrapolation of the completeness-corrected distributions down to the Galactic center. Consequently, they do not take into account depletion in the number of OCs by tidal disruption and enhanced frequency of collisions with molecular clouds in the inner parts of the Galaxy. In this sense, the above numbers of Galactic OCs must be taken as upper-limits.

For the completeness-corrected distribution function in the restricted zone (with a scale-height of $57 \mathrm{pc}$ (Table 1 ) and $\frac{\Delta \theta}{2 \pi}=$ $0.05)$ we derive $R_{\mathrm{D}}=1.4 \pm 0.2 \mathrm{kpc}$ and $\rho_{\odot}=795 \pm 70 \mathrm{kpc}^{-3}$ (Fig. 11). This number can be compared with that obtained assuming a uniform, cylindrically-symmetric distribution of OCs around the Sun. With a total number of OCs up to a distance from the Sun of $1.3 \mathrm{kpc}$ of 341 , and using a vertical height of $\approx 2 \times$ the scale-height we derive $\rho_{\odot}=281 \pm 16 \mathrm{kpc}^{-3}$, which corresponds to the observed OCs. Correcting for the average completeness in the restricted zone $(\sim 47 \%)$ produces a probable total of $\sim 730$ OCs, and a number density of $\rho_{\odot}=600 \pm 33 \mathrm{kpc}^{-3}$, which within the uncertainties roughly agrees with the value derived from the distribution function.

\section{Concluding remarks}

In the present paper we use a sample of 654 open clusters (typically Trumpler types I to III) with published parameters to derive statistical properties related to age, distance from the Sun, reddening, Galactocentric distance and height with respect to the Galactic plane.

The population of OCs younger than $200 \mathrm{Myr}$ distributes vertically in the disk following an exponential-decay profile with a scale height of $z_{\mathrm{h}}=47.9 \pm 2.8 \mathrm{pc}$. Clusters with ages in the range $200 \mathrm{Myr}$ to $1 \mathrm{Gyr}$ distribute vertically with $z_{\mathrm{h}}=$ $150 \pm 27 \mathrm{pc}$, while older cluster distribute nearly uniformly in height from the plane so that no scale height can be derived from exponential fits. The average scale height, considering clusters of all ages, is $z_{\mathrm{h}}=57.2 \pm 2.8 \mathrm{pc}$. The above OC scale heights are considerably smaller than those attributed to the thin disk $\left(z_{\mathrm{h}} \approx 0.6 \mathrm{kpc}\right)$ as derived by means of stars (e.g. de Boer et al. 1997; Altmann et al. 2004; Kaempf et al. 2005). We confirm previous findings that $z_{\mathrm{h}}$ increases with Galactocentric distance, being about twice as large in regions outside the Solar circle than inside it.

The asymmetry in the vertical distribution of OCs allowed us to derive the displacement of the Sun above the Galactic plane as $z_{\odot}=14.8 \pm 2.4 \mathrm{pc}$, which agrees with previous determinations using stars.

Sample completeness affects critically the detection particularly of low-contrast OCs internal to the Solar circle and those distant from the Sun. We simulate the effects of completeness in all directions and for any distance from the Sun assuming that the intrinsic distribution of the number of OCs with a given number of stars (above the background) measured in a 
restricted zone outside the Solar circle is isotropic. As a consequence we derived the radial dependence of completeness. The observed radial distribution of OCs in terms of Galactocentric distance does not follow the expected exponential profile, instead it falls off both for regions external to the Solar circle and more sharply towards the Galactic center. Correction for completeness produced radial distributions which agree with exponential disks throughout the Galactocentric distance range $5-14 \mathrm{kpc}$, with scale lengths $R_{\mathrm{D}}=1.5-1.9 \mathrm{kpc}$. These values of $R_{\mathrm{D}}$ are smaller than those implied by stars, which may reflect intrinsic differences in the spatial distribution of OCs and stars.

With respect to the vertical disk structure described by the OCs, we showed that older clusters distribute in exponential disks with larger scale heights than young OCs. In addition we confirmed previous observations that the scale height increases with Galactocentric distance. The latter result was further enhanced by the completeness-corrected $z$-distributions. The completeness-corrected OC scale height is comparable to those implied both by the $\mathrm{HI}$ and $\mathrm{CO}$ distributions.

We derived a number-density of solar-neighbourhood (with distances from the Sun $d_{\odot} \leq 1.3 \mathrm{kpc}$ ) OCs of $\rho_{\odot}=795 \pm$ $70 \mathrm{kpc}^{-3}$, which implies a total number of (basically Trumpler types I to III) OCs of $\sim 730$ of which $\sim 47 \%$ would already have been observed. Inferences on the total number of OCs in the disk can be made based on the local number density and radial/azimuthal completeness-corrected OC distributions. This estimate is important for the realization of what to expect in future surveys of the Galaxy. Direct extrapolation of the completeness-corrected distributions down to the Galactic center suggests that the total number of OCs in the Galaxy is in the range $(1.8-3.7) \times 10^{5}$. These numbers must be taken as upper-limits because the extrapolation does not take into account depletion in the number of OCs by tidal disruption and enhanced frequency of collisions with molecular clouds in the inner parts of the Galaxy.

The observed and completeness-corrected OC agedistribution functions can be described by a combination of two exponential-decay profiles characterizing the young and old OC populations with age scales of $\sim 100 \mathrm{Myr}$ and $\sim 1.9 \mathrm{Gyr}$, respectively. As a consequence, scenarios based on constant star-formation and OC-disruption rates do not apply to the data. Comparing the number of embedded clusters from IR studies in the literature and the present observed age-distribution function of very young OCs we derive survival rates in the range $3.4-8 \%$, in agreement with the estimates of Lada \& Lada (2003).

Acknowledgements. We thank an anonymous referee for interesting remarks. This publication makes use of the WEBDA open cluster database, as well as data products from the Two Micron All Sky Survey, which is a joint project of the University of Massachusetts and the Infrared Processing and Analysis Center/California Institute of Technology, funded by the National Aeronautics and Space Administration and the National Science Foundation. We acknowledge support from the Brazilian Institution CNPq.

\section{References}

Altmann, M., Edelmann, H., \& de Boer, K. S. 2004, A\&A, 414, 181 Bahcall, J. N., \& Soneira, R. M. 1981, ApJS, 47, 357

Bergond, G., Leon, S., \& Guibert, J. 2001, A\&A, 377, 462

Bica, E., \& Bonatto, C. J. 2005a, A\&A, 431, 943

Bica, E., \& Bonatto, C. J. 2005b, A\&A, 443, 465

Bica, E., Dutra, C. M., \& Barbuy, B. 2003a, A\&A, 397, 177

Bica, E., Dutra, C. M., Soares, J., \& Barbuy, B. 2003b, A\&A, 404, 223

Bica, E., Bonatto, C., \& Dutra, C. M. 2003c, A\&A, 405, 991

Binney, J., \& Tremaine, S. 1987, in Galactic Dynamics, Princeton series in astrophysics (Princeton, NJ: Princeton University Press)

de Boer, K. S., Aguilar Sanchez, Y., \& Altmann, M., et al. 1997, A\&A, 327, 577

Bonatto, C., Bica, E., \& Dutra, C. M. 2004, A\&A, 422, 555

Bonatto, C. J., \& Bica, E. 2005, A\&A, 437, 483

Chen, B., Stoughton, C., Smith, J. A. et al. 2001, ApJ, 553, 184

Cohen, M. 1995, ApJ, 444, 874

de Vaucouleurs, G., \& Pence, W. D. 1978, AJ, 83, 1163

Dias, W. S., Alessi, B. S., Moitinho, A., \& Lépine, J. R. D. 2002, A\&A, 389, 871

Dickey, J. M., \& Lockman, F. J. 1990, ARA\&A, 28, 215

Dutra, C. M., Bica, E., Soares, J., \& Barbuy, B. 2003, A\&A, 400, 533

Faber, S. M., Burstein, D., Tinsley, B., \& King, I. R. 1976, AJ, 81, 45

Friel, E. D. 1995, ARA\&A, 33, 381

de la Fuente Marcos, R. 1998, A\&A, 333, L27

Georgelin, Y. P., \& Georgelin, Y. M. 1970, A\&A, 6, 349

Girardi, L., Bertelli, G., Bressan, A., et al. 2002, A\&A, 391, 195

Hammersley, P. L., Garzón, F., Mahoney, T., \& Calbet, X. 1995, MNRAS, 273, 206

Heyer, M. H., Brunt, C., Snell, R. L., et al. 1998, ApJS, 115, 241

Janes, K., \& Adler, D. 1982, ApJS, 49, 425

Janes, K., \& Phelps, R. L. 1994, AJ, 108, 1773

Kaempf, T. A., de Boer, K. S., \& Altmann, M. 2005, A\&A, 432, 879

Kent, S. M., Dame, T. M., \& Fazio, G. 1991, ApJ, 378, 131

King, I. 1966, AJ, 71, 64

Knapp, G. R., Tremaine, S. D., \& Gunn, J. E. 1978, AJ, 83, 1585

Lada, C. J., \& Lada, E. A. 2003, ARA\&A, 41, 57

Lyngå, G. 1982, A\&A, 109, 213

Majewski, S. R. 1993, ARA\&A, 31, 575

McCuskey, S. W. 1969, AJ, 74, 807

Mermilliod, J. C. 1996, in The Origins, Evolution, and Destinies of Binary Stars in Clusters, ed. E. F. Milone, \& J.-C. Mermilliod, ASP Conf. Ser., 90, 475

Nilakshi, S. R., Pandey, A. K., \& Mohan, V. 2002, A\&A, 383, 153

Ortolani, S., Bica, E., \& Barbuy, B. 2005, A\&A, in press

Pavani, D. B., Bica, E., Ahumada, A. V., \& Clariá, J. J. 2003, A\&A, 399, 113

Platais, I., Kozhurina-Platais, V., \& van Leeuwen, F. 1998, AJ, 116, 2423

Reid, M. J. 1993, ARA\&A, 31, 345

Ruprecht, J. 1966, BAICz, 17, 33

Salpeter, E. 1955, ApJ, 121, 161

Schechter, P. 1976, ApJ, 203, 297

Skrutskie, M., Schneider, S. E., Stiening, R., et al. 1997, in The Impact of Large Scale Near-IR Sky Surveys, ed. Garzon et al. (Netherlands: Kluwer), 210, 187

Tadross, A. L., Werner, P., Osman, A., \& Marie, M. 2002, NewAst, 7 , 553

van den Bergh, S., \& McClure, R. D. 1980, A\&A, 88, 360 